Review paper

\title{
The advent of anthropomorphic three-dimensional breast phantoms for $\mathrm{X}$-ray imaging
}

\author{
Kristina Bliznakova \\ Department of Medical Equipment, Electronic and Information Technologies in Healthcare, Medical University of Varna, Varna, Bulgaria
}

\section{A R T I C L E I N F O}

\section{Keywords:}

Anthropomorphic breast phantoms

Computational phantoms

Physical phantoms

Tissue-equivalent materials

X-ray

Breast imaging

\begin{abstract}
A B S T R A C T
Anthropomorphic breast phantoms are both, computational and physical, anatomically and radiologically realistic models of the human female breast, and are considered an excellent tool for development, comparing, characterizing and optimizing of novel and existing breast screening and diagnostic modalities. Their role in the objective assessment of diagnostic task performance of breast imaging systems without conducting high cost and time-consuming clinical trials, as well as in developing and optimizing clinical protocols and image reconstruction algorithms is tangible increasing. The aim of this paper is to present a historical journey from the advent of the breast anthropomorphic models used with $\mathrm{x}$-ray modalities, their further development and realistic improvement, to current achievements. Anthropomorphic breast models are both computational and physical. Computational phantoms are mainly based on mathematical formulations and patient medical images. The mathematical breast models consist of mathematical description of the breast structures, while the patient-based are created from segmented clinical datasets acquired by dedicated breast Computed Tomography. The later approach guarantees a very high degree of realism. Molding techniques, 3D printing and low cost paper-based approaches are the main methods for creation of physical breast anthropomorphic models. Among them, 3D printing technologies provide an excellent opportunity to create realistic models of the breast by using a number of printing materials with x-ray characteristics similar to these of breast tissues. The anatomical and radiological realism of the anthropomorphic breast phantoms together with the possibility for unlimited exposure during important diagnostic tasks is transforming them into a key instrument of every Radiology Department.
\end{abstract}

\section{Introduction}

Anthropomorphic human phantoms are a key instrument in many tasks in Diagnostic Radiology. They permit unlimited exposure during important tasks, such as training activities, image quality assessment, patient dose optimization, clinical commissioning, which is in fact their strong advantage. In breast imaging, anthropomorphic models are used to validate, evaluate and optimise novel imaging techniques for screening and diagnosing the breast, as well as for systems' quality control, optimisation of clinical protocols and image reconstruction algorithms. During the last few years, they turned out to be the 'heart' of ambitious virtual clinical trials dedicated to objective assessment of diagnostic tasks performance of breast imaging systems [1-4], which may effectively replace the high cost and time-consuming randomized clinical trials.

Anthropomorphic breast phantoms are both, computational and physical models of the human female breast, and are considered an excellent tool for development, comparing, characterizing and optimizing of novel and existing breast screening and diagnostic modalities. Anthropomorphic phantoms are real or virtual objects, composed of tissue-equivalent materials that aim to provide a realistic and accurate representation of the anatomy and the properties of tissues, organs, whole body, for use in clinical and virtual studies carried out in research as well as in clinical daily tasks. In case of breast, realistic and accurate anatomy presentation means to mimic the breast outer shapes and the main internal tissue structures, the fibroglandular tissue, the adipose tissue and the various types of breast lesions for the diversity of breast types. The properties of the modelled structures depend on the tissue substitutes used in x-ray breast imaging, and specifically their elemental composition, physical density and effective atomic number [5].

Anthropomorphic breast models dedicated for x-ray imaging applications are both, computational and physical models of the breast. Computational phantoms are explicitly based, either on mathematical formulations, or patient medical images. Mathematical breast models consist of mathematical description of the breast structures. One

E-mail addresses: kristina.bliznakova@mu-varna.bg, kova@gmail.com. 
limitation of these models is the impossibility of encompassing the diversity of breast anatomical variations and their anatomical and radiological realism. To overcome this limitation, patient-based anthropomorphic breast phantoms with realistic tissue distributions are created from segmented clinical datasets acquired by breast CT, which guarantees a high degree of realism. While attempts to create these anthropomorphic computational breast phantoms date since the developing of the first stylized phantoms from 1960 [6-8], their physical realization has only recently become possible thanks to the advent of the $3 \mathrm{D}$ printing technologies. Molding techniques, 3D printing and low cost paper-based approaches are the main methods for creation of physical breast anthropomorphic models used with x-rays. Amongst them, 3D printing technologies provide an excellent opportunity to create realistic models of the breast by using a number of printing materials with $\mathrm{x}$-ray characteristics similar to these of breast tissues.

The aim of this paper is to present a historical journey from the advent of the breast anthropomorphic models used with x-ray modalities, their further development and realistic improvement, to current achievements. Both computational and physical breast models are considered as well as their role and use in interesting $\mathrm{x}$-ray breast imaging applications. The rest of the paper is structured as follows. In Section II, an overview of the breast anatomy and its radiological appearance is shortly described. The advances in anthropomorphic computational breast models are outlined in Section III, while in Section IV the physical realisation of the computational phantoms is concerned. Finally, conclusions are drawn on future developments of anthropomorphic breast models.

\section{Breast anatomy}

To model precisely the breast for use in x-ray imaging applications, extensive knowledge about its tissue structures, such as anatomical and radiological details, is needed. The female breast consists of glandular tissue of the tubuloalveolar type, connective tissue and other nonglandular tissue types: lymphatics, adipose tissue, blood vessels and nerves. Adipose and glandular tissues are the two predominant tissue types within the breast. The glandular tree consists of 15 to 20 irregularpyramidal shaped lobes as each lobe is drained by its own lactiferous duct, which is partitioned into narrowed and shorter branches, thus having a tree structure. At the suboreolar area the lactiferous ducts dilate to form the lactiferous sinuses (ampulla). At the ampullas, ducts of several lobes merge to form major ducts that emerge in the nipple independently, forming separate orifices on the nipple's surface. The ducts before the ampulla measure 1 to $2 \mathrm{~mm}$ in diameter while the major ducts diameter varies between 2 and $4.5 \mathrm{~mm}$. The total length of the ducts varies from 2 to $4.5 \mathrm{~cm}$. In the adult female breast, each lobe consists of several lobules. A lobule, 1 to $2 \mathrm{~mm}$ in diameter [9] is composed of ductules and alveoli that end at one common terminal duct. This structure called "terminal duct-lobular unit", is located at the endings of the tree branches. Both, ducts and lobules are embedded in loose connective tissue and are separated by dense connective tissue. Cooper ligaments are bands of dense connective tissue that support the breast, connecting the dermis of the skin with the deep fascia. They constitute fingerstall-like compartments. The lobes of the gland tissue and the adipose tissue are situated in these compartments [10].

Breast anatomy may be modelled precisely using either mathematical models of the breast structures or patient diagnostic images. Based on these computational models, three main approaches are exploited in manufacturing physical anthropomorphic breast phantoms: (a) by using molding techniques, (b) printing the different breast structures separately, followed by assembling the complete breast phantom, and (c) printing the breast model as a whole.

\section{Computational 3D anthropomorphic breast models}

The anthropomorphic computational breast models possess realistic
3D breast tissue distribution and anatomical features. In general, they can be classified as breast models based on applying mathematical techniques and generated from patient medical imaging modalities.

\subsection{Mathematical breast models}

Mathematical breast phantoms are based on modelling the elements of the breast, through mathematical techniques. The mathematical breast models represents in a complex way the external breast shape, mammary duct system, Cooper's ligaments, pectoral muscle, vessels, skin, mammographic texture, and breast abnormalities. This approach was chosen originally by Bliznakova et al. [11] and by Bakic et al. $[12,13]$, which both use a combination of solid-geometry and voxel based techniques to create a 3D anatomically realistic breast model, which is the main tool for generating synthetic mammograms that resemble real ones.

\subsubsection{UPenn anthropomorphic breast model}

In 2002, a research group from the University of Pennsylvania reported on the development of the first anthropomorphic computational breast phantom implemented through modelling of two ellipsoidal regions of large scale tissue elements: predominantly adipose tissue (AT) and predominantly fibroglandular tissue (FGT) $[13,14]$. These regions contain adipose compartments as well as breast ductal network, which are mathematically modelled by realistically distributed medium scale phantom elements: shells, blobs, and the simulated ductal tree. The ductal network model is implemented through Ramification matrices which describe the branching patterns of tree-like structures [12]. This duct network model was evaluated by comparing simulated trees with real ducts from galactogram images. A compression model required for the application of this model in 2D mammography and breast tomosynthesis (BT) studies is also developed based on tissue elasticity properties and a breast deformation model. Deformed slices are stacked to produce a model of the compressed breast. The adipose compartments are approximated by thin shells in the AT region and small blobs with varying size to allow for normal breast anatomic variations in the FGT region. The interiors of the shells and blobs have the elastic and x-ray attenuation properties of adipose tissue. The phantom at that stage of development did not include modelling of small-scale tissue structures. Further improvement concerned the realistic simulation of the breast adipose compartments and Cooper's ligaments, which is implemented by applying an iterative region-growing algorithm, initialized by selection of seed points [15]. A compartment is then grown from each seed, assuming an initial ellipsoidal shape. The growth of all compartments continues simultaneously until the user selected stopping criteria are met. Fig. 1 shows the comparison of two sections from the two versions of the UPenn software phantom. Further addition of small-scale features, generated by using power-low noise and the implementation of optimization algorithm for fast generation of high-resolution anthropomorphic breast phantoms with voxel size in the range of $25-1000 \mu \mathrm{m}^{3}$ / voxel [16], resulted in an improved version of this digital phantom [17].

Proposed algorithms, however, assumed each phantom voxel to contain single tissue type, which results in phantom projection images with notable artefacts near the borders between regions of different materials, particularly at the skin-air boundary. Several methods were proposed in order to overcome these limitations. One solution is to simulate smaller voxels. Reducing the voxel size, however, extends the phantom generation time and increases memory requirements. Another method is to account for the partial volume of various tissues in a voxel $[18,19]$. The $x$-ray linear attenuation coefficient of each voxel is calculated by combining attenuation coefficients proportional to the voxel sub-volumes occupied by the various tissues. This breast model showed to generate mammographic images with spectral power law exponents $(\beta)$, similar to those calculated from clinical images [20,21].

The UPenn anthropomorphic breast phantom is the key component in studies concerning 2D and 3D breast imaging techniques. Interesting 


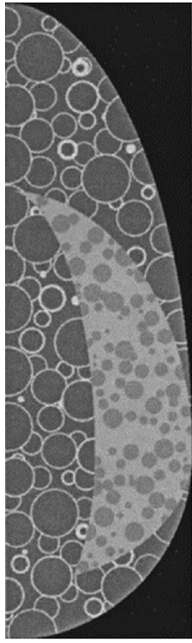

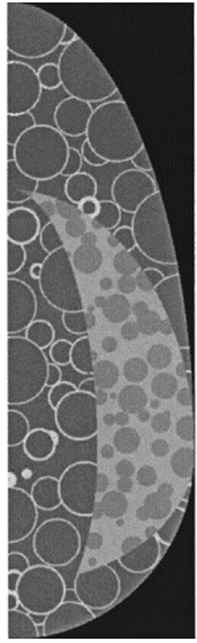

(a)
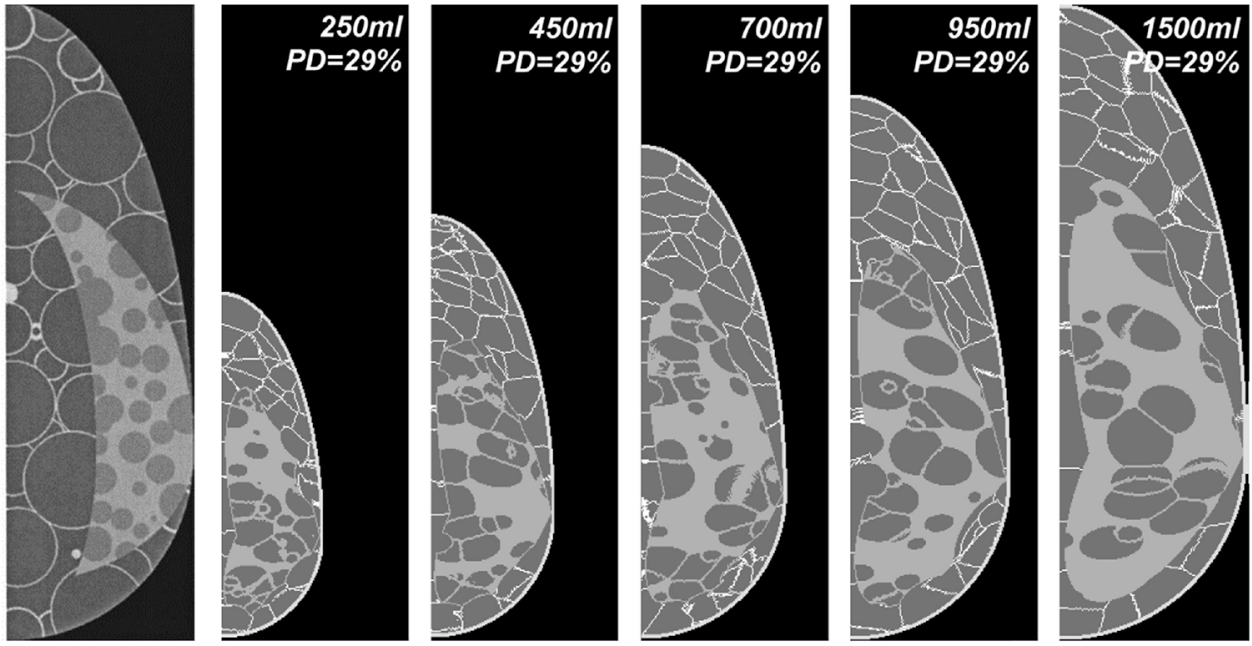

(b)

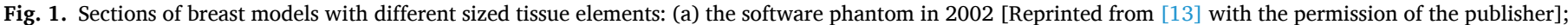
(b) the software phantom in 2011 [Reprinted from [15] with the permission of the publisher].

applications include the development of accurate breast dosimetry [22] and Monte Carlo method dedicated to mammography [23], evaluation of novel mammography noise reduction method [24], mammogram registration algorithm [25], analyzing texture properties of breast images $[26,27]$, assessment of various acquisition parameters on the mass detectability and reconstruction techniques $[28,29]$. This breast phantom is the basic component in conducting initial virtual clinical trials [30] as well as in the fabrication of physical breast models [31].

\subsubsection{UPatras anthropomorphic breast model}

In parallel, a research group from the University of Patras developed an anthropomorphic breast model which presents a complex aggregate of breast shape, duct system, Cooper's ligaments, texture and abnormalities as shown in Fig. 2 [11]. The process of building the breast phantoms starts always with modelling of the breast external shape, which defines the desired size and the shape of the breast, and which modelling is implemented as a combination of 3D two geometrical primitives: an elongated semi-ellipsoid and an elongated semihyperboloid. Ductal network is simulated using a network of

\section{Adipose compartments}

\section{Breast shape}
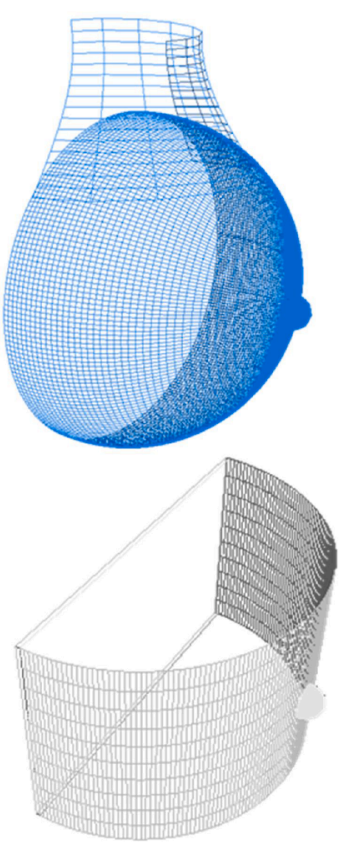
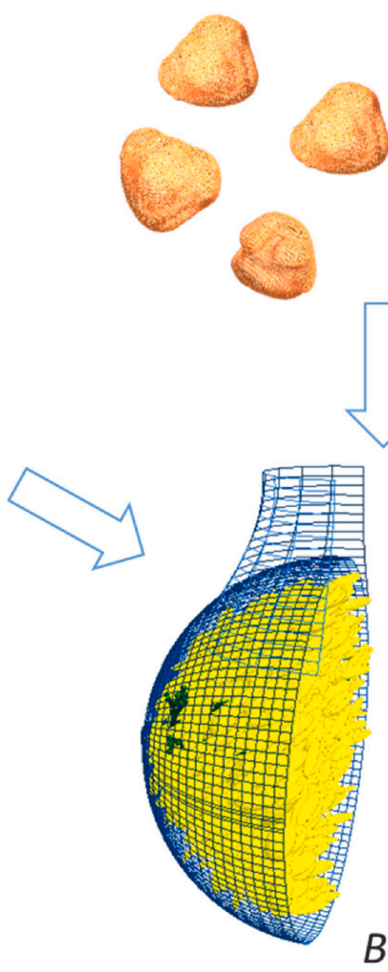

Fig. 2. Composing the 3D Upatras breast model.

\section{Breast model}
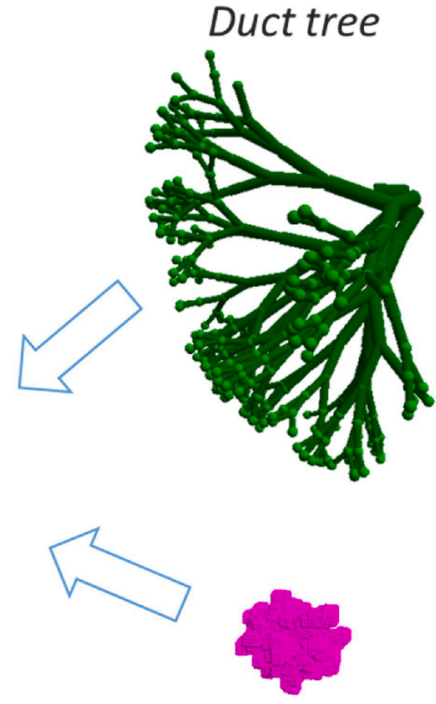

Abnormalities 

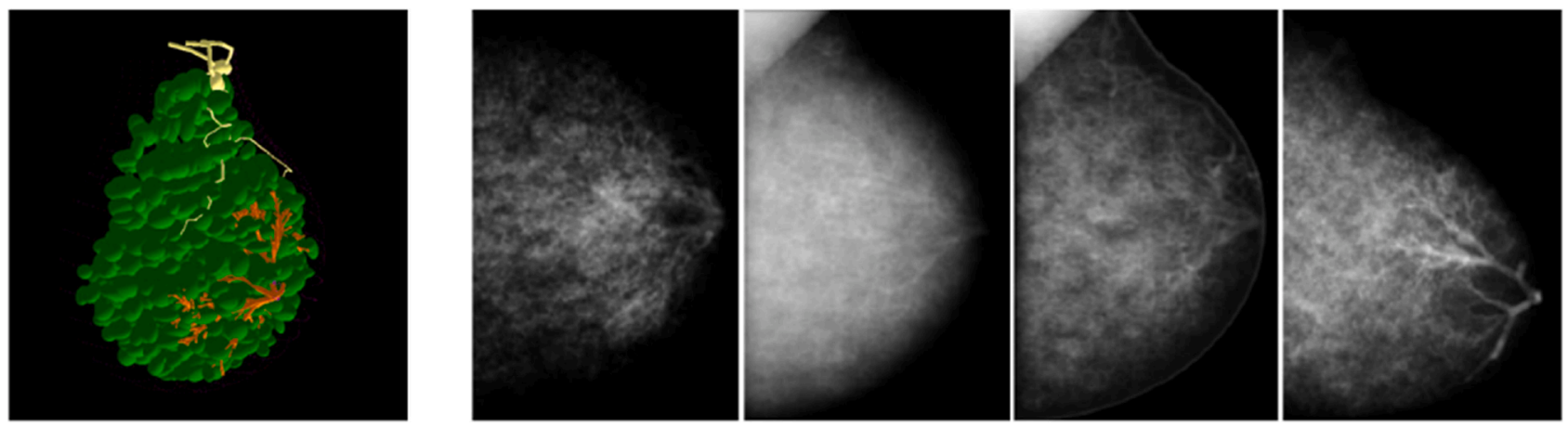

Fig. 3. Breast model (left) and four generated mammographic images (right). [Reprinted from [32] the permission of the publisher].

cylinders, probabilistically arranged in the breast as branches, in a treelike arrangement, starting from the nipple and restricted by the external breast shape. The 3D texture simulates the presence of adipose, fibrous and connective tissues as well as other non-glandular tissue types that are not explicitly modelled. A random walk based texture algorithm, followed by a series of 3D image processing is applied in order to obtain realistic 3D mammographic texture [32]. Cooper's ligaments are modelled as a set of thin ellipsoid shells, originating at randomly sampled positions in the breast model. Adipose compartments are represented by the volume enclosed by Cooper's ligaments. The pectoralis muscle is approximated as a cone shaped object, while the breast abnormalities are modelled with round, ovoid, elongated or irregular shapes. Models of irregular breast abnormalities are created either by using random walk algorithm [33] or based on segmented patient data $[34,35]$. Compression algorithm is also available with this breast phantom for mammography and BT applications [36].

Improvement of this breast model concerned realistic modelling of the adipose compartments. Segmented adipose compartments from patient CT images are available from Imran et al. [37]. The 3D models are represented in polygon-mesh form and additionally can be inserted within the breast model instead of using the ellipsoid adipose compartments [38]. To facilitate the comparison of results with other research groups, the compressed breast shape is also available as a combination of two geometrical primitives: a semi-cylinder to approximate the shape and a semi-ellipsoid to approximate the nipple. The UPatras model showed to generate mammography images which closely resemble visually and quantitatively the clinical mammograms.

There is a dedicated software application, the BreastSimulator [39], distributed to the scientific community, combining the above mentioned functionalities, which application appears to be a main instrument of the research group when $\mathrm{x}$-ray breast imaging research and training is considered [5]. This application is used in generation of anthropomorphic breast phantoms which were successfully evaluated for use in optimization of a dedicated breast CT scanner [40,41].

This digital model is mainly exploited in testing of novel breast imaging techniques such as breast dual energy imaging [42], phase contrast breast imaging, as well as use of monochromatic beams for lower dose breast tomosynthesis [43] and mammography detector performance [44]. The phantoms produced with the software are also used as computational prototypes for the manufacturing of anthropomorphic physical breast models, suitable for image quality assessment of 2D and 3D breast $x$-ray imaging systems $[45,46]$.

\subsubsection{FDA anthropomorphic breast model}

Based on these developments, a research group from FDA presented an advanced open source multimodal mathematical breast phantom to be used by the scientific community [47]. Breasts are generated from analytical expressions followed by voxel discretisation. Specifically, the shape of the breast surface is created by applying a series of transformations to a base superquadric surface. Then, a 1-mm skin layer and a nipple is added to the anterior side of the surface and a backing muscle layer is added to the posterior side. In the interior of the surface, glandular compartments are defined based on a random Voronoi segmentation. For each segmented glandular compartment, a ductal tree is grown using a random branching algorithm starting from the nipple. At the terminal branches of each ductal tree, terminal duct lobular units are added. Initially, the interior of the phantom is assigned to be purely glandular tissue. Random fatty lobules are inserted to create subcutaneous and perilobular fat layers along with some fatty structures within the glandular regions. Each fatty lobule is incased by a ligament structure [48]. The output of the model is a set of voxels of arbitrary size and each voxel contains a single type of tissue. Additional structures like blood vessels, chest muscle, and ligaments are further inserted (Fig. 4).

Compression of breast tissues, which is performed during mammographic examination is simulated by using the FeBio software package (www.febio.org). This requires generation of a tetrahedral mesh version of the breast phantom. Fat and glandular elasticity properties are assigned to each mesh element based on the local tissue components.

This advanced phantom is used in virtual clinical clinical studies that are promising approach for evaluating the safety and effectiveness of imaging technologies with much less burden than clinical trials $[1,3]$. The phantom is also a base for the creation of paper-based physical breast phantoms [49], and is use in evaluation of breast lesion detection $[48,50]$ and comparison of clinical breast imaging systems [51].

\subsubsection{OPTIMAM anthropomorphic breast model}

In parallel to the FDA development, Elangovan et al. [52] presented a novel method for generating quasi-realistic voxel phantoms, which method can simulate the compressed breast used in mammography and BT. The anthropomorphic breast models are produced using a biologically inspired approach whereby features and structures are extracted from BT image planes of real breasts and used to synthesize 3D breast structure. The simulation process starts by generating a high resolution empty breast volume surrounded by a skin layer which is then filled with voxels labelled with different tissue classes based on tissue structures extracted from BT images. The overall 3D shape is obtained from patient's BT via a thresholding technique. A $1.5 \mathrm{~mm}$ thick tissue layer is then added to the 'skin' surfaces of the breast outline to represent the skin layer. The interior voxels are labeled as adipose tissue. The glandular tissue is based on segments of glandular tissue extracted from patient's BT images using an adaptive seeded region growing method. A database of randomly selected glandular tissue fragments is then created and use to produce 3D glandular tissue matrix which is placed in the breast adipose tissue. Blood vessels and Copper's ligaments are modeled as a dilated 3D wireframe of linear structures which appear on patient BT images: 3-4 mm diameter for the blood vessel network, and to 1-2 $\mathrm{mm}$ in diameter for the Cooper's ligament network. Simulated mammography and BT images possess similar $\beta$ and fractal dimension and have very similar visual appearance to the clinical images. During the visual assessment, the observers had difficulty in distinguishing real 


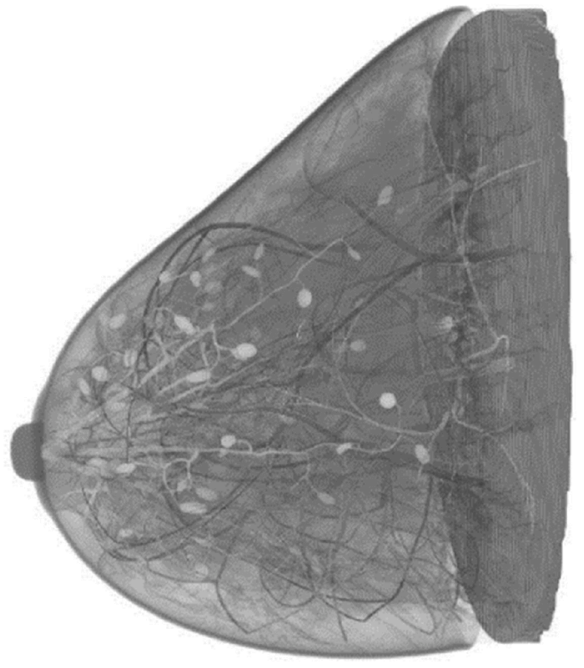

(a)

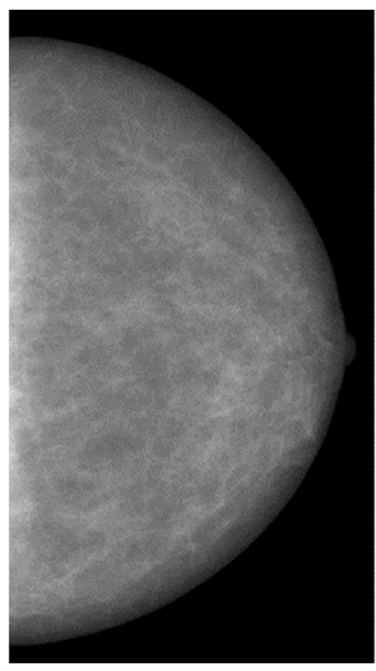

(b)

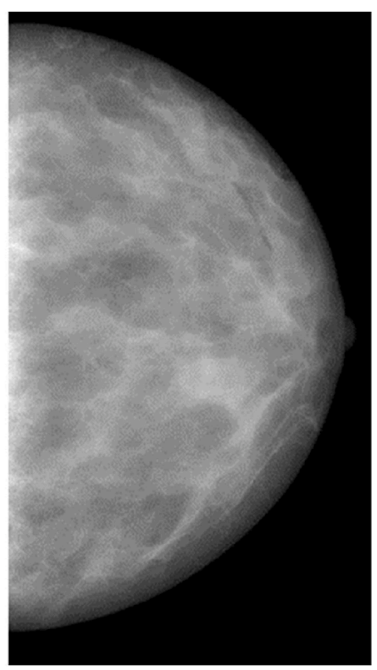

(c)

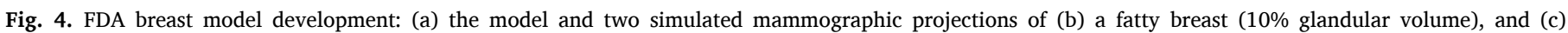

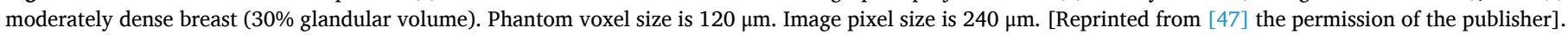

images from simulated images. The model is complemented by advanced spiculated computational tumour models [53,54], developed on a number of features, extracted from real spiculated tumour masses, needed to study the lesion detectability in breast images $[55,56]$ as well as to carry out virtual clinical studies [57] and comparison of different breast imaging techniques using human observation study [58].

\subsubsection{GE anthropomorphic breast model}

II et al. [59] proposed a mathematically defined solid 3D breast texture model inspired by the morphology of medium and small scale fibro-glandular and adipose tissue observed in clinical breast computed tomography images. The model is based on stochastic geometry theory used to mimic small and medium scale fibro-glandular and adipose tissue morphologies. Medium-scale morphology of each adipose compartment is simulated by a union of overlapping ellipsoids. To simulate the small scale texture aspect, small Voronoi cells with average volume of $0.5 \mathrm{~mm}^{3}$ are introduced at the boundaries of the ellipsoids, simulating irregular adipose compartment boundaries as observed in segmented clinical bCT datasets. The approach for generation of 3D breast texture models was evaluated in a two-alternative forced choice experiment, set to assess the visual realism of simulated BT reconstructed images. The percentage of correct answers was calculated as an indication for the realism of the simulated images from the texture model. This percentage varied between 65 and 82 for the four readers in the study. A quantification artifact, introduced by the size of the Voronoi cells at the transition of adipose/glandular tissue, was observed to reduce the realism of the texture model for higher density breasts. For almost entirely adipose breast types, the proposed model shows to simulate fairly realistic BT reconstructed images.

Based on the above methods and algorithms for creation of computational breasts, several research groups generated breast models for specific needs, extending the existing models to include improved and new models of anatomical structures $[60,61]$ and abnormalities such as irregular tumours and spiculated masses [62], as well as, with more functionalities such as suitable for multimodal image simulations [63]. One limitation of the generated mathematical anthropomorphic breast models is that although they claim that they encompass all possible breast densities, the visual presentation of simulated breast tissues on simulated mammograms does not encompass all clinical cases. Moreover, recent studies showed that characteristics of simulated x-ray images from these phantoms differ from these from patient images, for instance texture characteristics, statistical characteristics, "non-
Gaussian" statistics [64]. However, they are very useful in developing the initial concepts, testing hypothesis and designing clinical studies.

\subsection{Computational breast models based on patient medical data}

An attempt to include more realistic tissue distribution in breast models was approached by several authors [18,65-67] by exploiting conventional CT data sets. Three voxel breast models were created from segmented high-resolution CT data set of three patient breast specimens (taken from three different elderly women) which were fixated while being compressed [68]. These segmented breast models allowed more realistic representation of the glandular tissue and enabled reasonable calculations of the average glandular dose in mammography by Monte Carlo calculations as well as simulations of different imaging procedures.

A big step towards the development of computational anthropomorphic breast phantoms was made with the availability of dedicated breast CT (BCT) imaging system. The first such anthropomorphic phantom is reported by Li et al. [69] in 2009. A key step in generating of such breast models is the development of a methodology which concerns processing of the BCT slices and classification of breast tissues into adipose, fibroglandular and skin tissues, as depicted in Fig. 5a. The obtained simulated mammography image of the phantom is shown in Fig. 5b, demonstrating very realistic mammographic breast tissue appearing. The proposed methodology showed to possess some limitations in respect to the robust classification of high-resolution detail structures such as Cooper's ligaments, which were not fully visible on the simulated mammography image. To solve this limitation, the authors suggest to define these fine resolution structures mathematically and further to incorporate them into the computational breast volume. The approach of Li et al. guarantees a very high degree of realism; however, it represents a single breast composition and lacks flexibility to cover wide anatomical variations. In order to cover wide range of anatomical variations, Hsu et al. [70,71] developed a computational technique applied to the available limited number scans of human breast subjects [69] to generate a large number of computerized anthropomorphic breast phantoms. The technique that is based on morphing and deformation, demonstrated its ability to generate realistic anthropomorphic breast phantoms, which has been validated as well as visually by the involved radiologists.

To speed up the development of breast models based on patient BCT data, accurate anatomical characterisation of the breast with this 


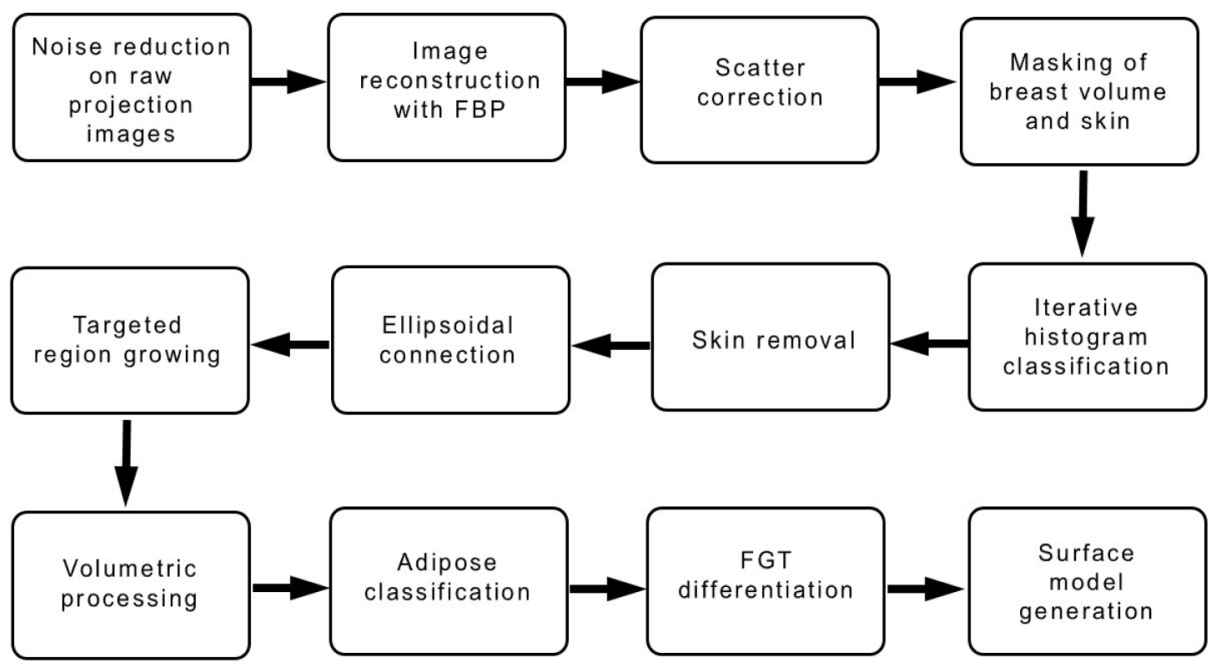

(a)

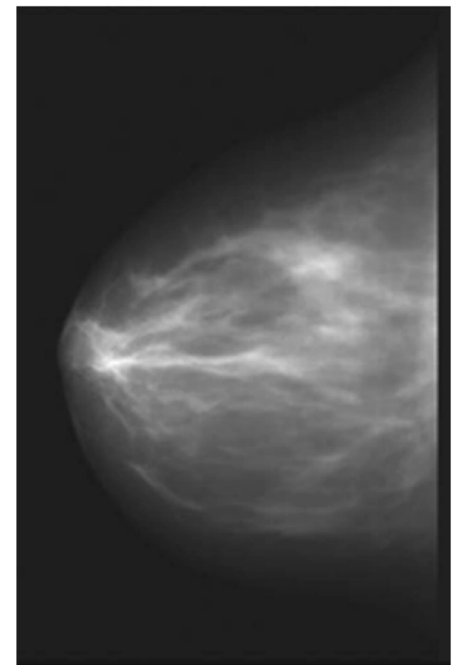

(b)

Fig. 5. Computational breast models based on patient medical data: (a) Methodology for generating 3D computerized breast phantom from empirical data based on work of Li et al. [69] and (b) A mammogram of computational breast model, based on patient BCT data [Reprinted from [69] with the permission of the publisher].

modality was needed. A number of breast anatomical metrics were introduced and characterized in detail by Huang et al. [72], describing the breast shape, breast diameter and length, breast glandular fraction in three breast regions, as a function of patient age and bra cup size. This was performed on the largest available set of images (219) acquired with BCT at that time. Based on these images, a research group from Duke University produced 224 virtual breast phantoms [73,74]. The authors also developed a finite element application to simulate different compression states of the breast, making the phantoms applicable to multimodality imaging research.

Based on the original scans by Huang et al. [72], in 2019, the Napoli group created anthropomorphic breast models [75-77]. The segmentation of the breast tissue types in BCT images is realised by a Matlab subroutine, exploited to classify BCT voxels into adipose tissue, fibroglandular tissue and skin. The classification algorithm is based on adaptive thresholding coupled to morphology processes such as erosion and dilation, opening and closing procedures.

Eighty eight computational breast phantoms were derived and used in evaluation of glandular dose distributions and imaging data. An example is shown in Fig. 6 for building a breast phantom for mammography applications. These breast models contained realistic glandular tissue distribution, which permits to validate conventional homogenous breast models against a consistent cohort of patients. Thus, these models contribute to an improved model that more accurately calculates the mean glandular dose in the breast during x-ray breast imaging [76-78] as well as for BCT optimization via virtual clinical trial [79], carried with patient-derived models rather than based on anatomical assumptions as in VICTRE project [2]. Similar approach to the approach of Napoli group was proposed by Garcia et al. [80].

\section{Clinical CT scan}

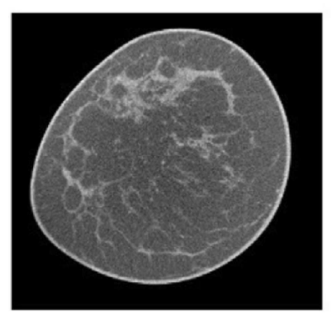

Tissue
classification
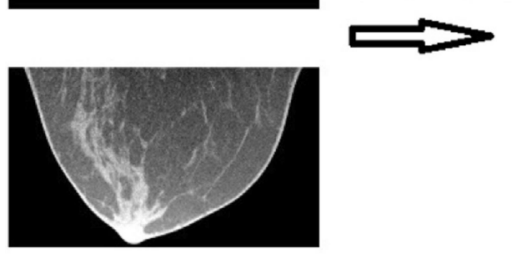

\section{Digital breast phantom for mammography and DBT}
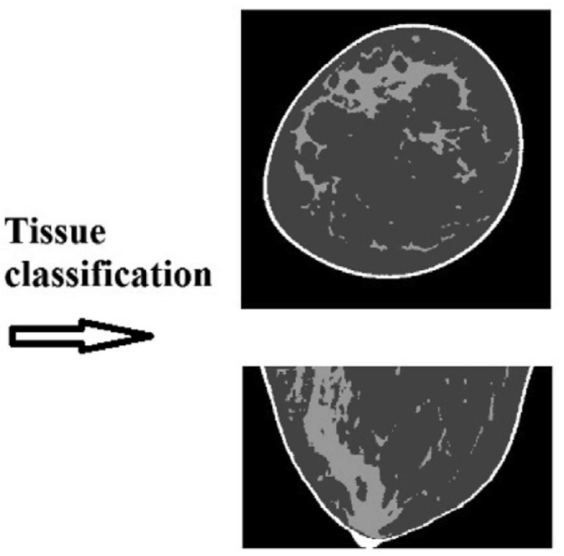

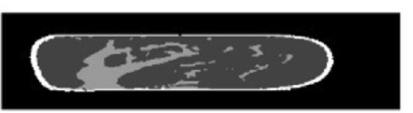

Digital

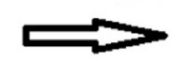
compression

$\square$ Skin $\square$ Adipose $\square$ Air $\square$ Glandular

Fig. 6. Example of coronal and sagittal views of a clinical BCT scan acquired at UC Davis (left), uncompressed digital breast phantom produced by means of tissue classification (center) and compressed digital breast phantom produced by means of the digital compression procedure (right). [Reprinted from [75] with the permission of the publisher). 


\section{Physical 3D anthropomorphic breast models}

The design and the realization of anthropomorphic physical phantoms had been pushed out when 3D printing technologies have been developed and introduced to the medical community in recent years. 3D printing technology provides the possibility to select amongst different printing techniques and new materials with characteristics (density, elemental composition, $\mathrm{x}$-ray properties) similar to these of human tissues, thus resulting in a successful 3D printed radiological model of the breast. The anthropomorphic physical phantoms for $\mathrm{x}$-ray applications replicate the shape, the anatomy of internal structures and x-ray characteristics of human tissues. In fact, their basic function is to mimic the radiological behavior of the real human tissues when exposed to x-rays. In this way, they can be used in experimental tests instead involving patients and thus pushing research and innovative developments in the field of Diagnostic Radiology. Thus, these physical models must be built from materials which elemental compositions are similar to these of the human tissues. In case of breast, the considered tissues are adipose, glandular, skin and tumor tissues.

The fabrication of physical anthropomorphic breast phantoms involves two processes: creation of a digital breast model, by using one of the approaches discussed in the previous section and the use of this model to produce the physical breast model. Anthropomorphic breast phantoms are manufactured generally in three ways: (a) by using molding technique, (b) printing the different tissues separately, followed by assembling the whole phantom and (c) printing the whole phantom. Enormous efforts are placed towards the development of new materials, because of the limitations of the ones currently available in terms of desired x-ray properties [81-83]. These efforts are related to sample preparation and full characterization in respect to elemental composition and physical properties. An extensive review of materials used in manufacturing of anthropomorphic models is given in details in [5], as some key materials used for manufacturing $\mathrm{x}$-ray breast phantoms are shown in Table 1.

\subsection{Breast phantoms based on molding techniques}

The CIRS BR3D (CIRS, Inc., Norfolk, VA) mammography phantom is fabricated in D-shaped slabs, which are stacked in order to produce the complete phantom as shown in Fig. 7. Each slab contains two tissue equivalent materials mimicking $100 \%$ adipose and gland tissues together in an approximate $50 / 50$ ratio by weight. The two tissue

Table 1

Key materials, used in manufacturing the breast phantoms for use in mammography diagnostic range.

\begin{tabular}{lllc}
\hline $\begin{array}{l}\text { Approximated } \\
\text { tissue }\end{array}$ & Material & $\begin{array}{l}\text { Density } \\
\mathrm{g} \mathrm{cm}^{-3}\end{array}$ & References \\
\hline Adipose & $\begin{array}{l}\text { Ultra-high-molecular-weight } \\
\text { polyethylene }\end{array}$ & 0.94 & {$[84]$} \\
& ABS (Acrylonitrile Butadiene & 1.02 & {$[81]$} \\
& Styrene) & & \\
& PE-12 & N/A & {$[85]$} \\
& Clear resin & 1.18 & {$[86]$} \\
& QuickWater & 1.02 & {$[82]$} \\
& Paraffin & 0.93 & {$[81]$} \\
Glandular tissue, & 20\% PVAL gel & 1.19 & {$[87]$} \\
& Water & 1.00 & {$[88]$} \\
& Nylon & 1.11 & {$[81]$} \\
& Flex & 1.14 & {$[81]$} \\
& TangoBlackPlus & 1.11 & {$[82]$} \\
& VeroClear & 1.18 & {$[82]$} \\
& VeroWhitePlus, TangoPlus & N/A & {$[89]$} \\
& Polylactide doped with Cu and & & \\
& W & & {$[90]$} \\
& Polyvinyl alcohol cryogel & N/A & {$[81]$} \\
& Grey resin & 1.175 & {$[91]$} \\
\hline
\end{tabular}

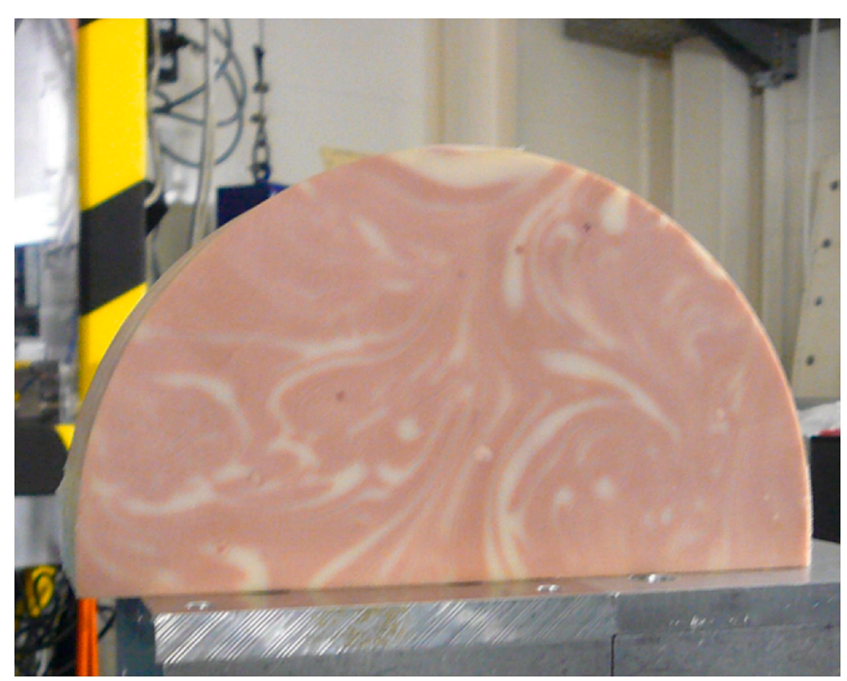

Fig. 7. CIRS BR3D mammography phantom, designed to assess detectability of various size lesions within a tissue equivalent, complex, heterogeneous background.

equivalent plastic materials are "swirled" together to create a heterogeneous structure. Normally, several slabs are manufactured that can be rearranged in different orders to easily obtain different but limited number of backgrounds. One of the slabs contains an assortment of microcalcifications, fibrils and masses.

The phantom is used in image quality and research studies involving BT and breast CT techniques; with a major advantage of providing a more complex background. The resulted structures on a mammography image, although with fuzzy borders, appear not as realistic as these observed in patient images. For example, tiny structures, like the Cooper's ligaments, cannot be fabricated by using this production technology and thus these structures will not be present on x-ray images. A recent BT study reported by Sage et al. [92] showed that the textured background of the BR3D is highly contrasted and this results in highcontrast generated artefacts over the entire phantom. Further, on BT images, the circular structures, in which the inserts are placed, were clearly visible, which affected the image score measurements and analysis.

An anthropomorphic breast phantom, dedicated to both x-ray and MRI breast imaging modalities was developed by Freed et al. [93] by mixing fresh egg white and melted refined lard, and placing the obtained mixture in a jar with a breast-like shape. This phantom proved to be a useful tool for quantitative assessment of image quality in 2D and 3D $\mathrm{x}$ ray techniques, for detection and characterization purposes (Fig. 8). The proposed method is limited to modelling large structures in the phantom that appear considerably larger than those in the patient data set. Further, there is no established methodology for the production of phantoms with a desired glandularity.

Breast molds were also used by Ruvio et al [90] for the creation of multimodal breast phantoms dedicated to $\mathrm{x}$ ray-based, MRI and ultrasound imaging techniques. The manufacturing process included five stages using three different breast molds: an external breast mold, skin mold and internal fibroglandular mold. The external breast mold replicated the shape of a human breast in a prone position, obtained from MRI patient data. The main element in the production of the skin element is polyvinyl alcohol cryogel. The adipose structure is presented by a mixture of water and a beeswax mixture, while the agar-based fibroglandular, tumor and pectoralis muscle components as a mixture of the liquid components (water, glycerol and benzalkonium chloride) and the dry components (agar, $\mathrm{SiC}, \mathrm{Al}_{2} \mathrm{O}_{3}$ ). Current limitation is related to the impossibility of compressing the phantom due to the fact that the fat-mimicking material is not elastically compressible. This results in a poor contrast between tumor and fibroglandular tissue. Although the 


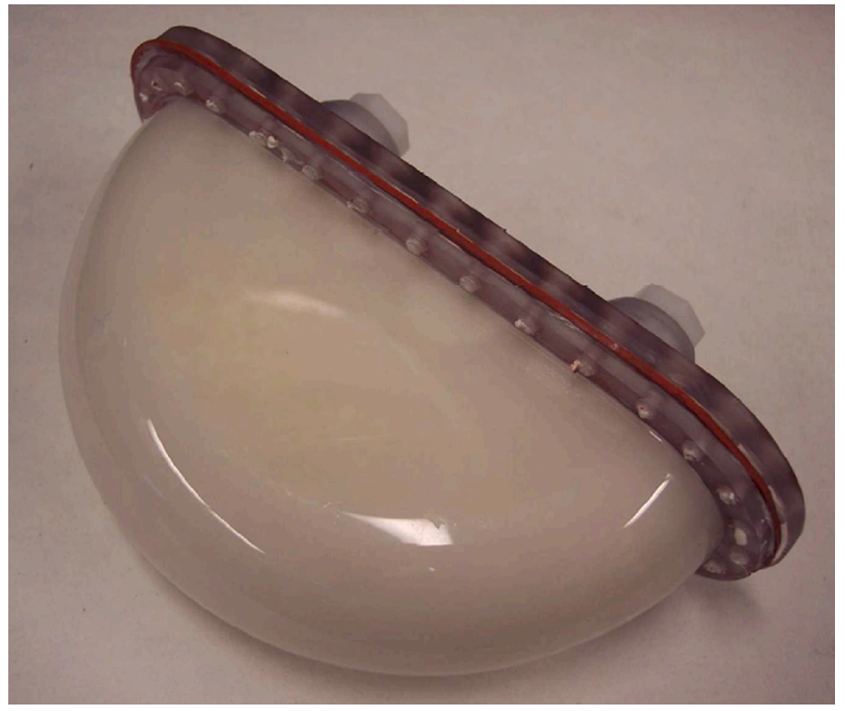

Fig. 8. A photograph of a physical anthropomorphic compressed breast phantom. [Reprinted from Freed et al [93] with the permission of the publisher].

challenging work to fabricate tissues that mimic the important imaging parameters for the three modalities, a good agreement between reference and measured properties was demonstrated in the study. This type of phantoms is dedicated for use in multimodality cross-calibration and training where human subjects and cadavers cannot be exploited.

\subsection{Sphere breast phantoms}

This is a specific case of the approach discussed in the next subsection. Several anthropomorphic breast phantoms are constructed by combining a homogeneous background with spheres of different dimensions made of tissue equivalent material [94-97]. They are widely used for evaluating the image quality and doses in mammography, and also for optimizing the examination procedures of new mammographic techniques, such as dual-energy mammography, contrast-enhanced dual-energy mammography, BT and BCT. In 2010, Gang et al. [98] showed that equal volumes of differently sized acrylic spheres provide a fractal dimension of 3 as well as a power law exponent $\beta$ equal to 3 .
Based on this development, a team from the Department of Radiology, Catholic University of Leuven developed a sphere phantom consisting of an acrylic container filled with acrylic spheres and water (Fig. 9a). The two main parts of the breast phantom are the acrylic semi-cylinder container, which approximates a compressed shape breast of thickness of $58 \mathrm{~mm}$ and equal volumes of acrylic spheres of six different diameters [99]. As an alternative to spheres placed in air, the space between the spherical objects can also be filled with water, a material with $\mathrm{x}$-ray properties close to these of the real breast tissue. It has been evaluated on a Siemens mammographic system with tomosynthesis option, showing results for the power law exponent in the range of the exponents measured in patient data. Although the image pattern achieved with these phantoms is not similar to a real breast image, the sphere phantom is very simple to construct and can easily produce different background realizations.

This physical phantom is characterized with air bubbles which were visible at the upper side of the phantom because of the practical difficulties in completely filling the phantom with water. The improvement of this phantom is related to the use of computational phantom, which is a flexible approach in optimising phantom content [100-102].

Similar approaches were reported by Bliznakova et al [46,103,104], where semi-cylindrical containers printed from epoxy resin or nylon are filled with spheres of epoxy resin, while the filling material is paraffin or animal lard. Fig. 9b shows a small size breast phantom, whose shape is a semi-cylinder and is manufactured from White resin. In this physical phantom, twenty seven spheres, manufactured from Grey resin, with a radius ranging between $6 \mathrm{~mm}$ and $13 \mathrm{~mm}$ are placed, followed by adding animal fat. It was used recently in studying phase-contrast breast imaging [103] and for developing a channelized model observer (CHO) for use in quality control of BT imaging systems [105].

\subsection{Assembled breast phantoms from separately printed elements}

This approach requires the availability of computational models of the main breast type tissues: skin, glandular, adipose tissues and breast lesions such as microcalcifications and masses. These separate computational models are generated either based on mathematical approaches implemented through dedicated computer programs [11,41] or are based on segmentation algorithms applied to patient medical images [66]. Every modelled breast tissue is saved in a different file and further processed to $s t l$ format for 3D printing. An example for the realization of physical breast phantom using this approach is shown in Fig. 10a, where the modelled computational elements of the breast, shown in Fig. 2, are
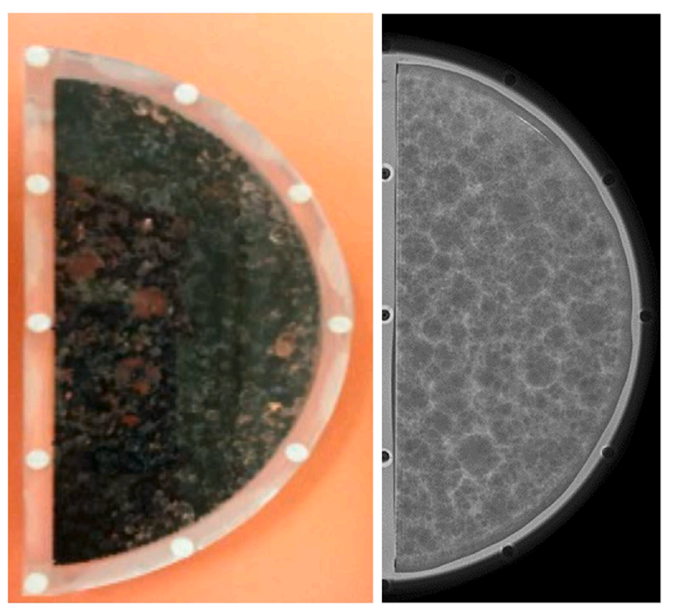

(a)

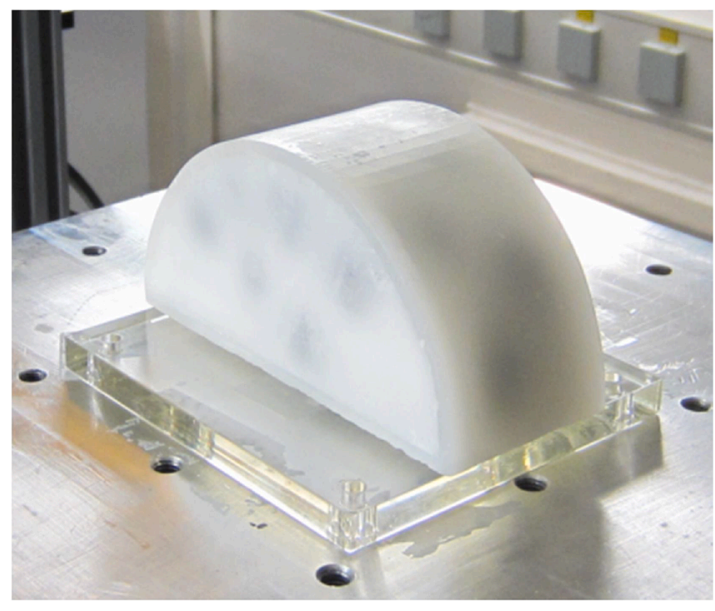

(b)

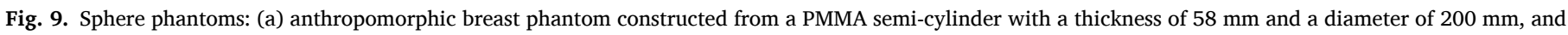

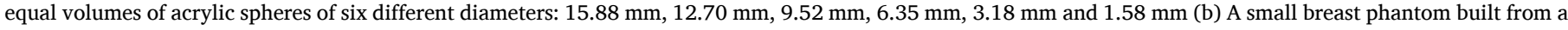
semi-cylinder container with a height of $40 \mathrm{~mm}$, a radius of $50 \mathrm{~mm}$, and $3 \mathrm{~mm}$ wall thickness filled with spheres manufactured from Grey resin. 


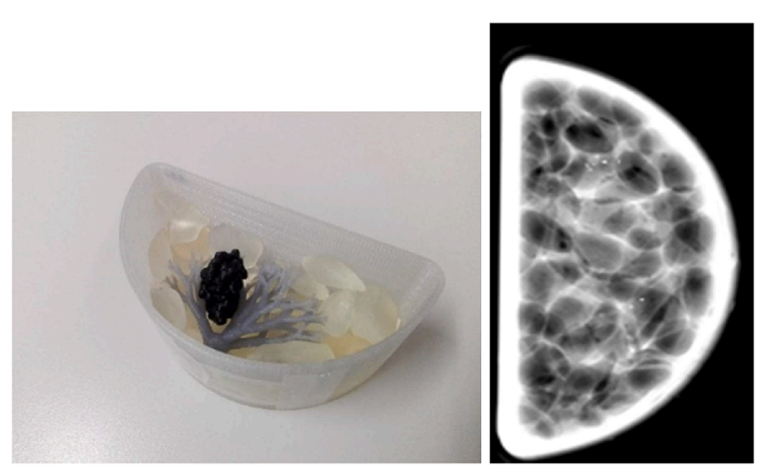

(a)
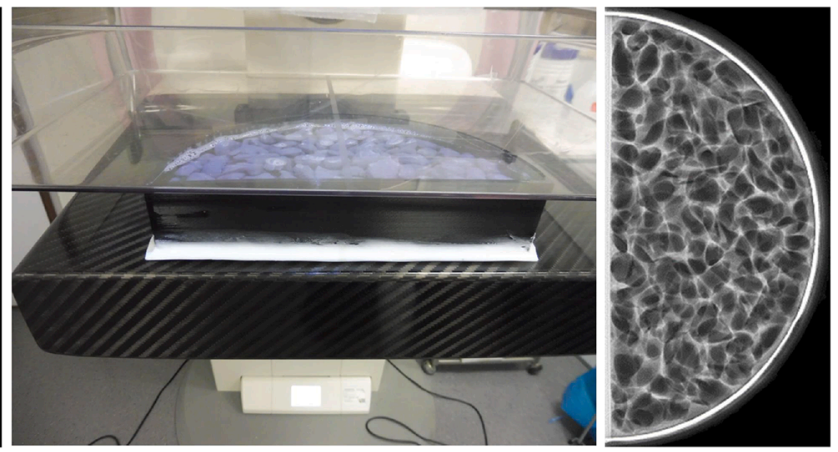

(b)

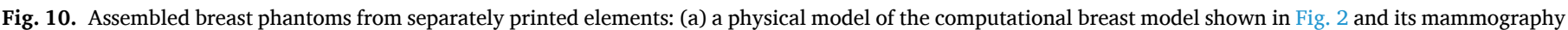
image; (b) improved physical breast model of the sphere physical phantom shown in Fig. 9a and its mammography image.

fabricated by using two 3D printing technologies. Stereolithography (SLA) is used to print the external shape, glandular tree and lesion, while fused deposition modelling (FDM) with ABS (Acrylonitrile Butadiene Styrene) filament is exploited for the adipose compartments [38,46]. The decision for using these printing materials is based on an extensive experimental study $[81,83]$. X-ray image of this breast model filled with water is shown in Fig. 10a, showing the realistic appearance of the microcalcifications, mimicked by crushed into a fine powder eggshells, while the breast mass is not visible. More research is needed towards novel 3D printing materials which will be able to mimick the x-ray properties of all breast tissue types.

In 2016, a collaborative work between researchers from three universities on improving the sphere phantom, shown in Fig. 9a, resulted in a new modified version, shown in Fig. 10b [106]. Models of manually segmented breast adipose compartments from CT images of a mastectomy specimen [66] were printed with a stereolithographic 3D printer using Formlabs Clear Resin. Further, they were placed in a semicylindrical container, $48 \mathrm{~mm}$ thick, filled with water, followed by a mammography image, obtained at Siemens Mammomat (Fig. 10b, right image). The evaluated experimental images showed a power law exponent higher $(\beta=3.8)$ compared to the original sphere model ( $\beta=$ $2.8)$ and for patient mammograms $(\beta=3.4)$. Further modifications of the compartment model aim at improving the agreement with the patient data which may be solved with the use of less dense printing material in order to increase the contrast of the compartments, inclusion of smaller compartments by downscaling the existing models, and refining the compartment segmentation method. Recently, another version of the sphere phantom from Fig. 9a was designed by a team from the University of Vienna, which model includes spheres, manufactured from VeroClear, while paraffin oil is used as filling material [107].

\subsection{Printing the whole breast phantom}

\subsubsection{Printing with one material}

Mathematical breast models are a data source used to manufacture 3D anthropomorphic physical phantoms for image quality assessment of 2D and 3D breast X-ray imaging systems. The physical UPenn phantom [31] is based on the mathematical breast phantom described in previous section [12-14]. The chosen manufacturing technology requires initially segmenting the voxels of the digital phantom in two components: (a) fibroglandular tissue and (b) adipose tissues. The former is produced with a polyjet Eden $500 \mathrm{~V}$ used with a tissue-equivalent material with $50 \%$ glandular equivalence (FC-720 photopolymer). The printing is performed in layer by layer to allow access to the empty spaces, which

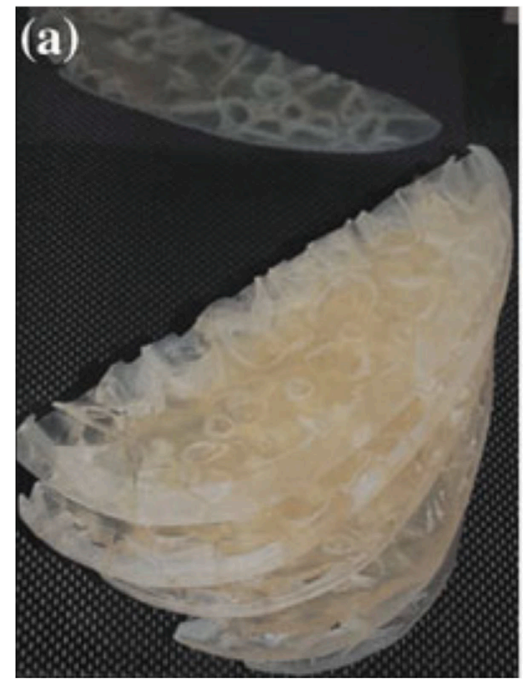

(a)

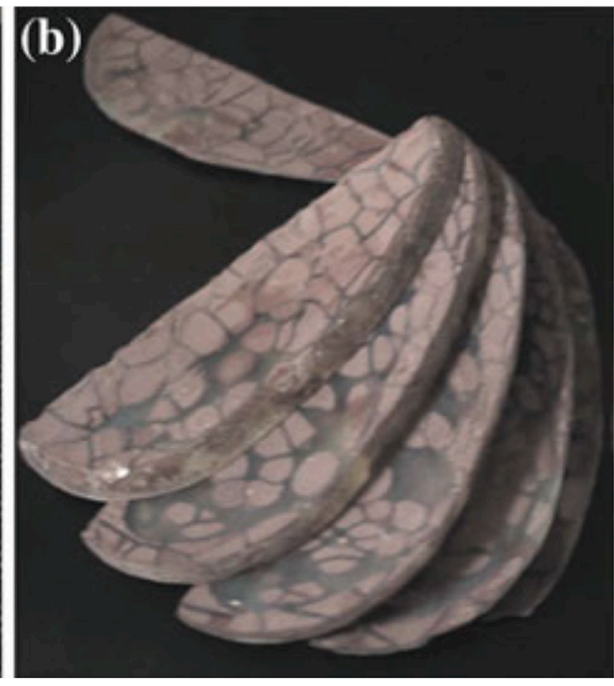

(b)

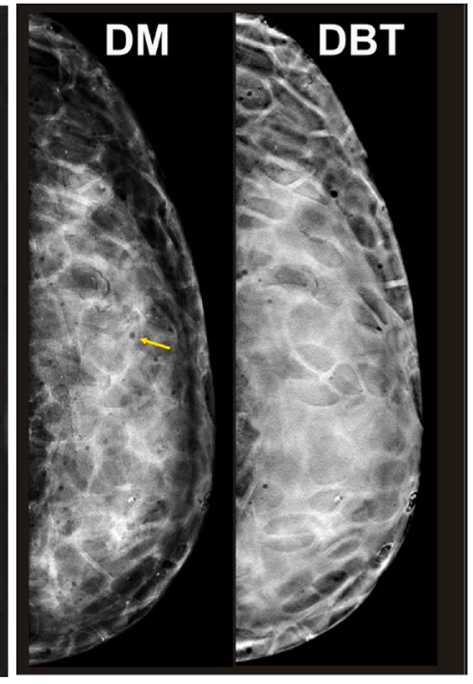

(c)

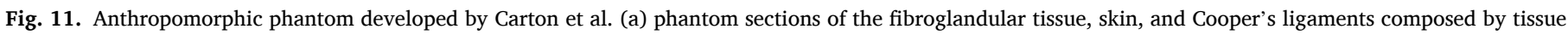

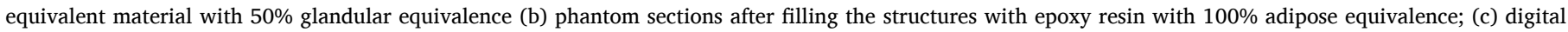
mammography (DM) image and reconstructed BT image of the prototype phantom shown in b. [Reprinted from [31] with the permission of the publisher]. 
are then filled with an adipose-equivalent. The sections are printed using a voxel resolution of $60 \mu \mathrm{m}$. Fig. 11a shows printed slices which represent the glandular part of the breast, while Fig. 11b depicts the complete slices with realized adipose compartments. The slabs are then combined together to create the final anthropomorphic phantom. The initial experimental evaluation of mammography and BT images of this physical phantom (Fig. 11c) reveals its potential to be used for both, qualitative and quantitative performance assessments for $2 \mathrm{D}$ and $3 \mathrm{D}$ breast $\mathrm{x}$ ray imaging systems. The mammography images obtained with this phantom are visually similar to clinical images. Some of the limitations of this technology are related to the non-fuzzy borders of the structures, the presence of residual air bubbles that are visible on the radiographs, long production time and high production costs.

Similarly, Mainprize et al. [85,108] reported on a two-component full-sized physical breast phantom. The digital voxel-based phantom [59] is converted to a stereolithography mesh-data format and inserted into a mesh-format ' $D$-shaped' compressed breast shell and further separated into four slabs to accommodate different inserts at different heights within the phantom. For each slab, the fibroglandular component is removed, leaving only the adipose component. The obtained adipose slab is then printed by using selective laser sintering printer set to $100 \mu \mathrm{m}$ resolution and polyamide-12, which have linear attenuation coefficients across an energy range of $15-40 \mathrm{keV}$ matching adipose tissue to within $10 \%$ to $15 \%$ equivalent volumetric breast density. Evaluated power spectral parameter $\beta$ was in the range between 2.3 and 3.6, defined by calculations on 2686 mammograms. Moreover, phantom measurements demonstrated a good agreement with similar excess entropy to that of the clinical full-field digital mammograms.

Breast Computed Tomography image set of a specific patient is the base for implementing a technique to generate a two-compartment anthropomorphic breast phantom by Prionas et al [88]. The glandular part is comprised of water, while the adipose part is composed of polyethylene. 3D images are initially acquired at a dedicated BCT, followed by noise removal and tissue segmentation in adipose and glandular components. The manufacturing process differs from the above mentioned as the phantoms are fabricated with water-jet computer numerical controlled machine. The stack of breast segments (Fig. 12a, b) was machined from $1.59 \mathrm{~mm}$ thick ultrahigh molecular weight polyethylene sheets using a $0.2 \mathrm{~mm}$ wide water-jet with the tool path centerline defined at the tissue structure edges. Then, the machined breast segments were stacked as shown in Fig. 12c. An outer container was molded around the breast phantom using thermoplastic to approximate the thickness of breast skin. The container holding the stack of breast segments was filled with water such that the air gaps in the phantom, representing the glandular tissue compartment, were filled with water.

This phantom demonstrated inplane similarity between the original patient images and BCT images of the breast phantom. Larger bodies of glandular tissue closely matched the original patient images. Advantage of the model is its modular design which allows for the insertion of other objects into any point in the glandular tissue compartment. Major limitations are related to both the technology and material used, resulting in adding length. The tolerance in the thickness of the polyethylene stock as well as the gaps between the produced breast segments resulted in $2.6 \mathrm{~cm}$ longer breast phantom compared to the original patient's breast. The material is polyethylene and this limits the application of the phantom to $\mathrm{x}$-ray modalities where compression to the breast is not applied. Other minor issues are related to the presence of air-bubbles in the glandular tissue, which bubbles may be removed by using more sophisticated degassing techniques during phantom assembly. Another minor issue is related to the water-jet cutting process, which may also result in a reduction of the outer adipose edge and expansion of the glandular tissue structure edges.

Patient-based two dimensional mammographic images are the key

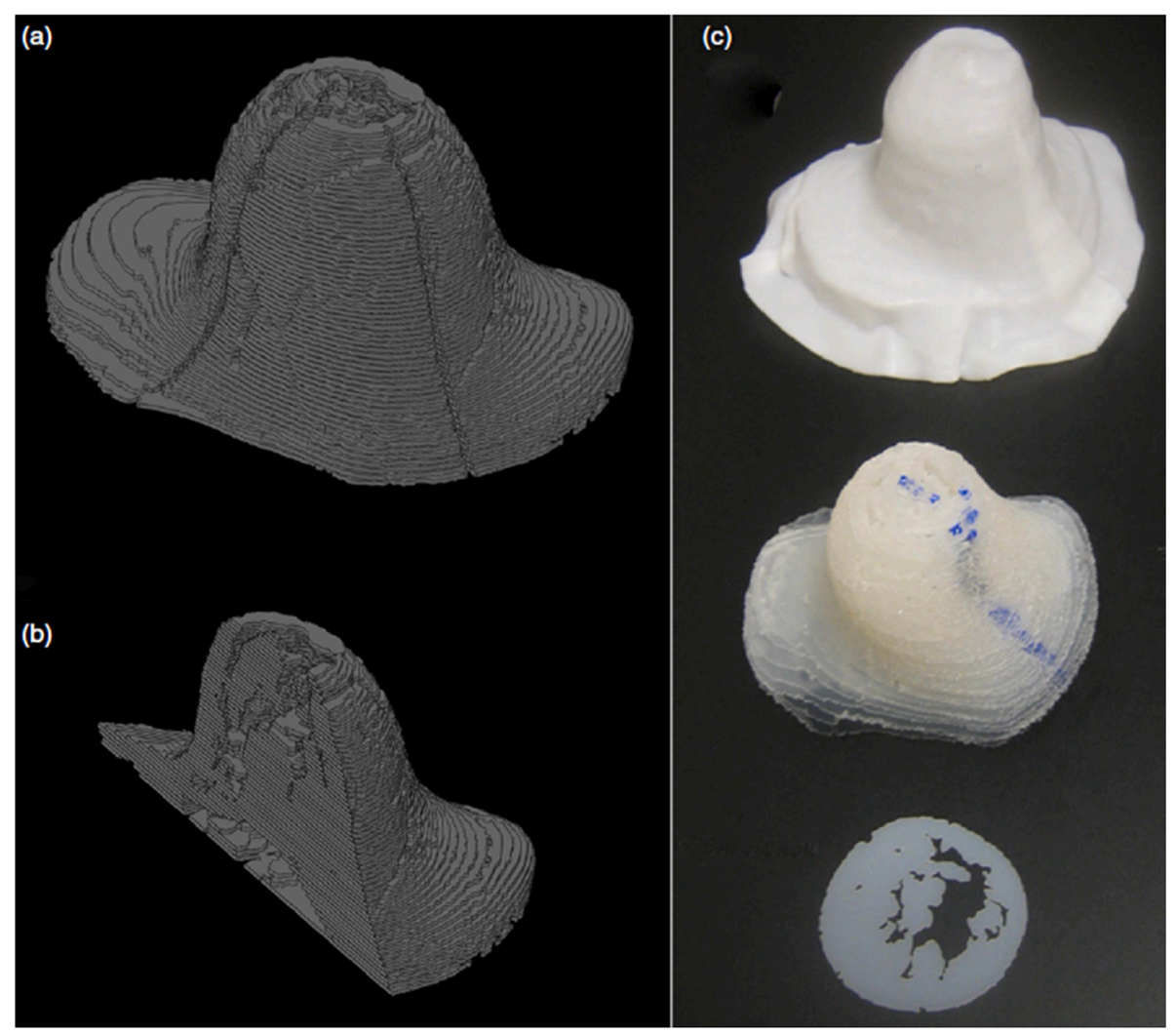

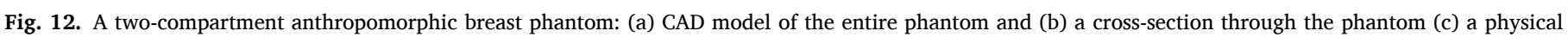

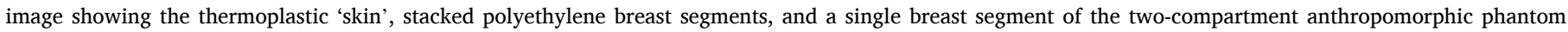
[Reprinted from [88] with the permission of the publisher]. 
source in a methodology for printing physical anthropomorphic breast phantoms, proposed and accomplished by two research groups [109,110] in 2018 and 2019. Badal et al. [110] developed an easily reproducible methodology for fabrication of anthropomorphic breast models based on 2D mammography image. An inkjet-based Objet260 Connex3 printer with VeroMagnenta and VeroCyan printing materials were used for the fabrication of the breast objects. The choice of this printing technology is well justified in an extensive experimental study which involved three different printers based on different technology: stereolithography, fused deposition technology and inkjet [111]. Fig. 13 shows an example of cranio-caudal mammogram from a patient with dense breast who was diagnosed with invasive ductal carcinoma; the corresponding computational model created by a dedicated Python open-source script (mammoreplicator), the physical realization of the phantom, and an x-ray projection of the phantom acquired in a clinical mammography system.

The key element in this approach is the mammoreplicator, which calculates the thickness of the printed material as a function of each image pixel grey level and the differences in x-ray attenuation between breast tissues and 3D printing materials. Each pixel in the image is transformed into a column of the appropriate height via the mammoreplicator.

The model was printed within $10 \mathrm{~h}$. It weighs $750 \mathrm{~g}$ and corresponds to a material consumption of $\$ 220$. It is currently validated to correctly reproduce the radiological properties of the mammary gland in mammography. A study of the similarity of the original and phantom mammograms demonstrated that the anatomical features were reproduced with good fidelity. Main limitation is related to the resolution of the obtained phantom. Although the pixel resolution of the patient mammogram was $100 \mu \mathrm{m}$, the 3D printer was not able to accurately replicate details in the clinical mammograms below $300 \mu \mathrm{m}$. The authors suggest to use this technology for the development of collections of representative patient models that could be used to assess the effect of anatomical breast variability on system performance, hence making bench testing studies a step closer to clinical trials.

Schopphoven et al. [109] used a patient mammography image from a breast compressed to a thickness of $32 \mathrm{~mm}$. The key procedure is related to the determination of the pixel intensities of the unprocessed images for varying printing material (polypropylene like printing material) thicknesses for the spectrum of the clinical image. This is realized by scanning of printed slabs with different thickness (up to $40 \mathrm{~mm}$ ) at clinical mammography unit. The relation between slab thickness and pixel intensity on mammography image is determined by using a 2nd degree polynomial model to fit $\ln \left(\mathrm{I}(\mathrm{z}) / \mathrm{I}_{0}\right)$ versus the slab thickness. Further, by using the inverse function of the fit model, and by using $\ln \left(\mathrm{I}_{\mathrm{x}}\right.$, $\mathrm{y}$, clinical $/ \mathrm{I}_{0}$, clinical) as input for the inverse function, the authors generated a height 2D map from the clinical mammogram. The corresponding attenuation at pixel position $\mathrm{x}, \mathrm{y}$ of the clinical image is simulated by differences in height of the printing material, resulting in a relief-like structure on top of the phantom as shown in Fig. 14a.

Printing time of the phantom was about $11 \mathrm{~h}$ with a material consumption of $791 \mathrm{~g}$ of RGD450 ${ }^{\mathrm{TM}}$ and $31 \mathrm{~g}$ of support material. The discussed approach allows producing anthropomorphic phantoms for mammography that realistically simulate the anatomy and attenuation characteristics of a female breast and can be used for a variety of quality control and system optimisation tasks as well as for educational and scientific purposes. Current major limitations are related to the slightly reduced resolution seen in the depiction of small details in respect to the initial clinical image. This is due to the current chosen combination of printer and material (PolyJet printer in combination with the polypropylene-like printing material), which limits the printable structures size to about $200 \mu \mathrm{m}$. Structures are located on top of the phantoms, thus are positioned higher above the detector than the structures in the patient breast. This may result in stronger unsharpness in mammography images of thicker breasts due to geometrical magnification.

Simulated microcalcification clusters are inserted in this phantom using a 3D printed $4 \mathrm{~mm}$ base plate with movable inserts and crushed eggshells [112]. The base plate and inserts were printed using Objet30 Pro PolyJet ${ }^{\mathrm{TM}}$ 3D printer and the material VeroClear RGD810 (Stratasys, Eden Prairie, MN, USA). The base plate contained three circular cavities with a diameter of $60 \mathrm{~mm}$, into which separately modelled rings, numbered " 1 " to " 3 " as shown in Fig. 14b. The first ring, the "lesion ring", contains three circular cavities with a diameter of $10 \mathrm{~mm}$ and matching covers to enclose and seal the simulated microcalcifications. The other two rings are modelled solid and contain no structures. The three rings are designed with the same dimensions which means that they are interchangeable, the lesion positions can be varied relative to the anthropomorphic phantom over a wide range. Crushed eggshells were inserted into the cavities of the lesion ring and closed with a cover. Different microcalcification clusters are simulated via different amount and grain size of the eggshells.

An advantage of this approach is the modular design including the simple and cost-effective possibility to integrate further layouts of the

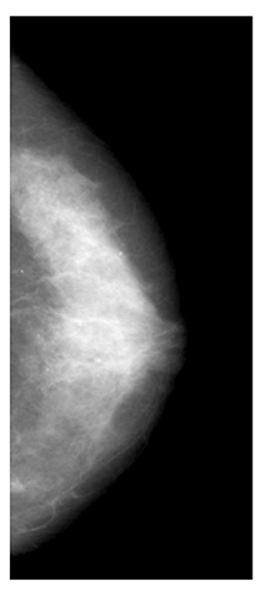

(a)

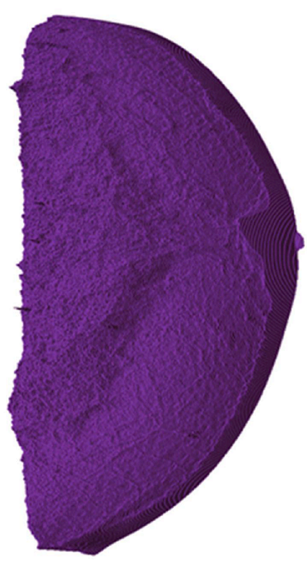

(b)

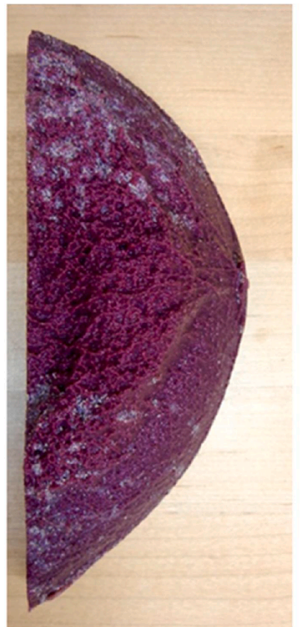

(c)

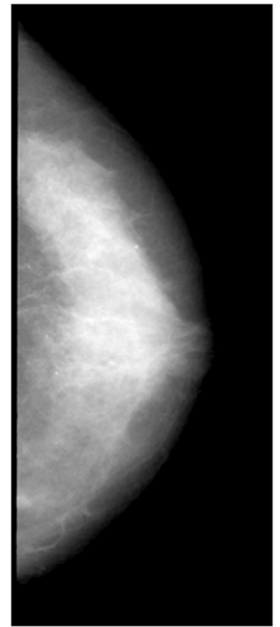

(d)

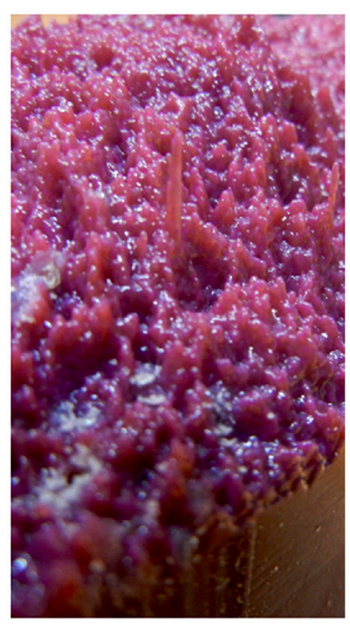

(e)

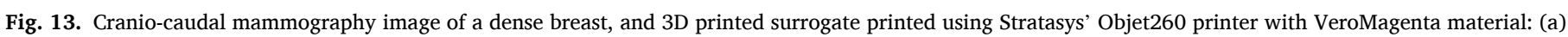

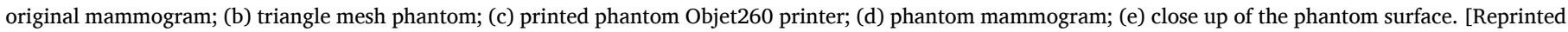
from [110] with the permission of the publisher]. 


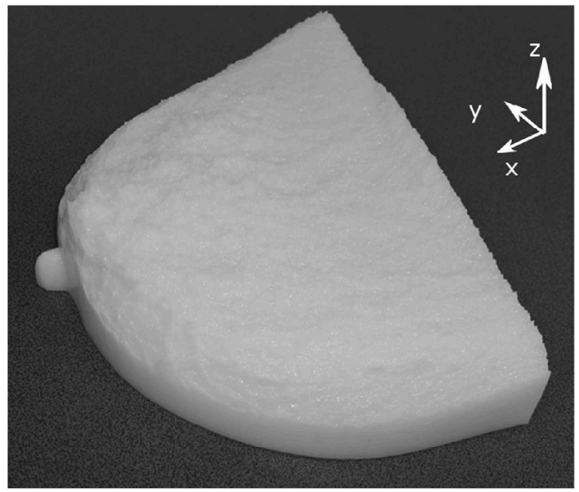

(a)

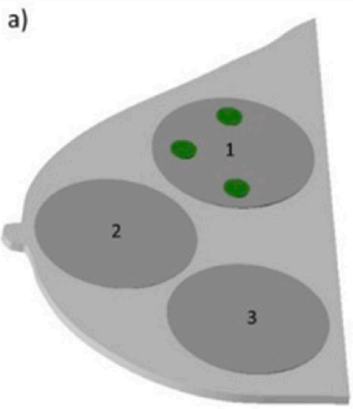

(b)

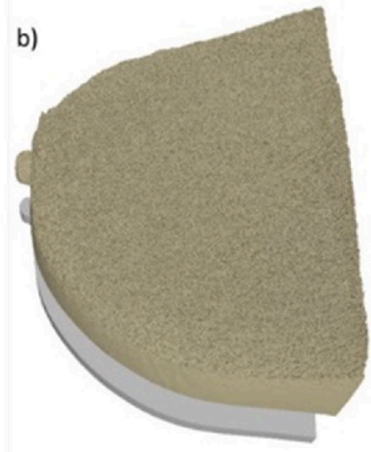

(c)

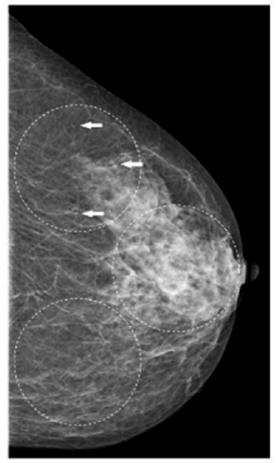

(d)

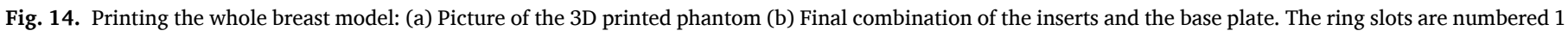

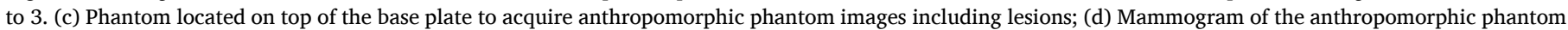

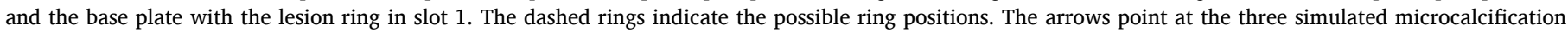
clusters of the lesion ring. [Reprinted from [112] and [109] with the permission of the publisher].

base plate and corresponding rings with different lesions, depending on the use. The phantoms fabricated with this technique are intended for use with 2D mammography. For 3D breast imaging, it is crucial the physical breast phantoms to mimic the spatial distribution of different breast tissues in three dimensions which may be completed by one of the approaches discussed below.

In 2018, Okkalidis et al. [113] suggested a new methodology for printing breast phantoms all at once directly from patient CT data by using FDM technology by varying the infill density of some uniform infill pattern. The pattern is used to accurately mimic the internal structure of the modeled 3D object. The thickest parts of the object are produced with maximum infill density of $100 \%$, while softer and lighter tissues are printed with a lower density. This allows both realistic and radiologically suitable fabrication of different tissue types. The method is based on reading the Hounsfield Unit value from each voxel and based on this value, the PLA (Polylactic acid) filament extrusion rate is controlled to produce the required amount of extruded filament. This method was applied in printing anthropomorphic breast phantom directly from patient BCT data [114]. Fig. 15a shows part of the printed phantom. The initial phantom evaluation at a conventional CT facility demonstrated visual similarity between original patient and phantom BCT slices (Fig. 15b, c), which motivates the team towards the development of a dedicated phantom for BCT studies and specifically in setting a future experimental setup for an accurate BCT dosimetry. Main limitations are the long printing time (several days) and the visible printed pattern due to the FDM technology used in the study.

\subsubsection{Printing with two or more materials}

Ideally, the use of different printing materials to reproduce the woman breast 'all in one' would result in the ideal anthropomorphic breast phantoms. A great step towards the realization of this goal has been made in 2015 by Kiarashi et al. [86], who managed to fabricate an anthropomorphic phantom of a compressed breast through 3D printing by using two printing materials in parallel. Segmented BCT images of a virtual breast phantom [115] were compressed [71] and the resulting breast model was produced by 3D printing after a careful selection of the printing material. Two anthropomorphic breast models were printed using an Objet500 Connex 3D printer. The first phantom, called Doublet, was printed by using two materials simultaneously: one for glandular and one for adipose tissue (Fig. 16a). Materials used to print the fibroglandular part were TangoGray and VeroWhite (mimicking 64\% glandular tissue), while TangoPlus, the least radiographically dense material available corresponded to $36 \%$ glandular tissue at $28 \mathrm{kVp}$ with $\mathrm{W} / \mathrm{Rh}$. The second phantom, called Singlet, was printed with the material that is used to print the glandular tissue in the first phantom (Fig. 16b). Half butter/half lard, beeswax, resin, olive oil were studied as filler materials

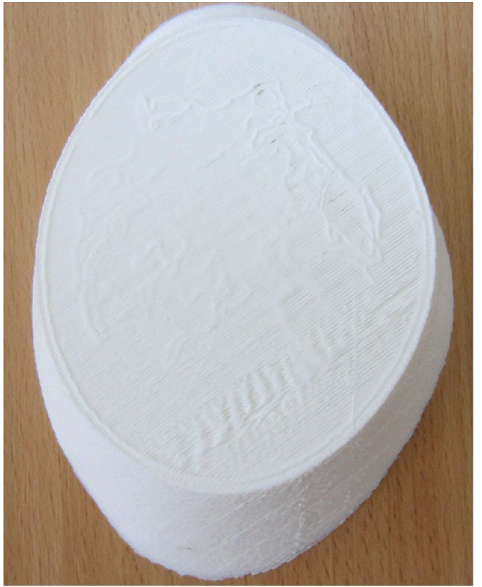

(a)

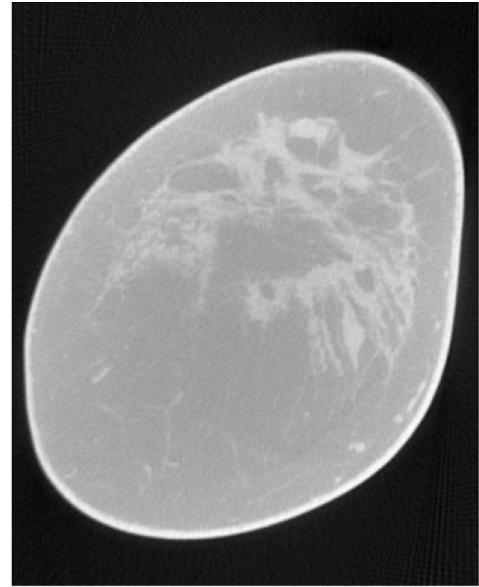

(b)

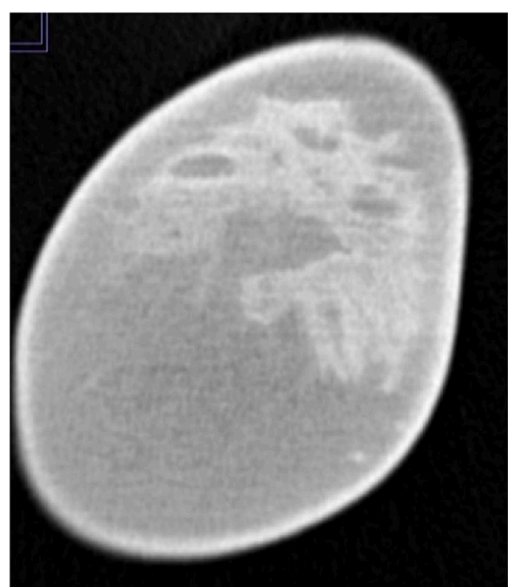

(c)

Fig. 15. Printing the whole breast model with one material: (a) A photo of the fabricated phantom and scanned at clinical CT, (b) original slice from patient BCT and (c) the corresponding slice of the scanned physical breast, shown in (a). 

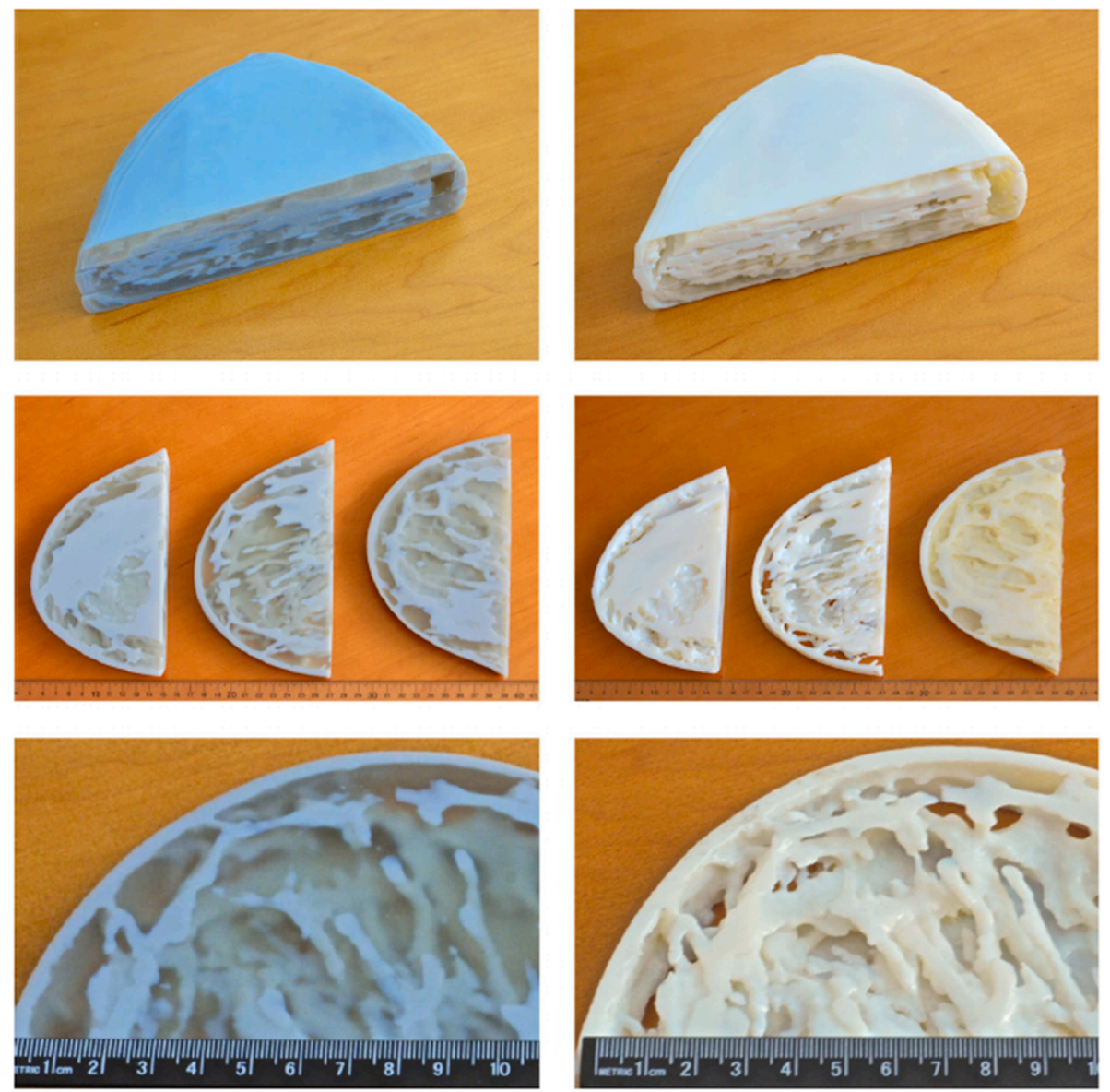

(a)

(b)

Fig. 16. Fabricated physical breast phantoms, the Doublet (a) with two materials and the Singlet (b) with a single material, both in three slabs of $15 \mathrm{~mm}$ each. [Reprinted from [86] with the permission of the publisher].

which are approximating attenuation coefficient of the adipose tissue. The resin approach was repeated several times using different filling techniques, but always resulted in some amount of undesirable air bubbles.

The physical phantoms demonstrated realistic breast anatomy radiographic appearance in both, 2D and 3D images. The power-law description of the mammograms of the physical phantoms was in good compliance with real human mammograms $[116,117]$. Limitations of the phantoms are related to the limited dynamic range and contrast imposed by the lack of suitable printing materials, a challenge that is to be solved with the availability of more materials. Though the limitations in the contrast, the Doublet phantom offers the key advantage of being an "all in one" design where the phantom (in whole or subsections) can be fabricated simultaneously in one pass.

Further evolution of the above approach in printing antropomorphic breast models to mimic patient anatomy in order to evaluate clinical mammography and digital breast tomosynthesis performance is reported by Rossman et al [118]. They created a modular phantom with an anthropomorphic region to allow for improved lesion and calcification detection as well as a uniform region to evaluate standard quality control metrics. The commercial product VeroPureWhite and the custom, tungsten-doped Jf Flexible resin were used to print the fibroglandular tissue, while the commercial TangoPlus and the third party Jf Flexible resin without dopant were used to represent low-density adipose tissue. The phantom was designed as two butterflied halves, however the design can be changed into a single breast volume for ease of handling or more thinner slabs to provide greater customization. The design includes possibility to add masses, iodinated masses, and calcifications. One of the current limitations of this phantom is that volumetric breast density equivalent below $36 \%$ cannot be achieved. Efforts are focussed on achieving low attenuation by trying different techniques.

In 2019, the Napoli group [4,91] used for the first time FDM 3D printer for manufacturing physical phantoms of both uncompressed and compressed breasts, mimicking the shape, as well as the anatomical and the radiological properties of real breast tissues. They used digital BCTbased phantoms and three different printing materials to fabricate a physical anthropomorphic breast phantom. PVA was used for printing the skin, while ABS and Nylon were used for printing the adipose and glandular tissue, respectively. The skin tissue was printed separately, since the computational breast phantoms are made of three material substitutes, corresponding to adipose, glandular and skin tissues, and the available Ultimaker $3 \mathrm{FDM}$ printer prints objects with a maximum of two materials. The later is represented as a PVA envelope (Fig. 17b) which encloses the breast inner volume represented by ABS and Nylon tissue substitutes. The models have a $100 \%$ infill density in order to reduce as much as possible the air gaps. The internal region of the breast can be represented either as printed slices of $10 \mathrm{~mm}$ in thickness or a single block of two-component material. Moreover, the authors designed a region in the printed slices to place TLD chips for dosimetric purposes as well as dedicated holes for placing the tumor printed lesions. These models were used in validation of Monte Carlo simulations [79,119].

\subsubsection{Paper-based breast anthropomorphic models}

A low cost approach in producing breast phantoms is using office 


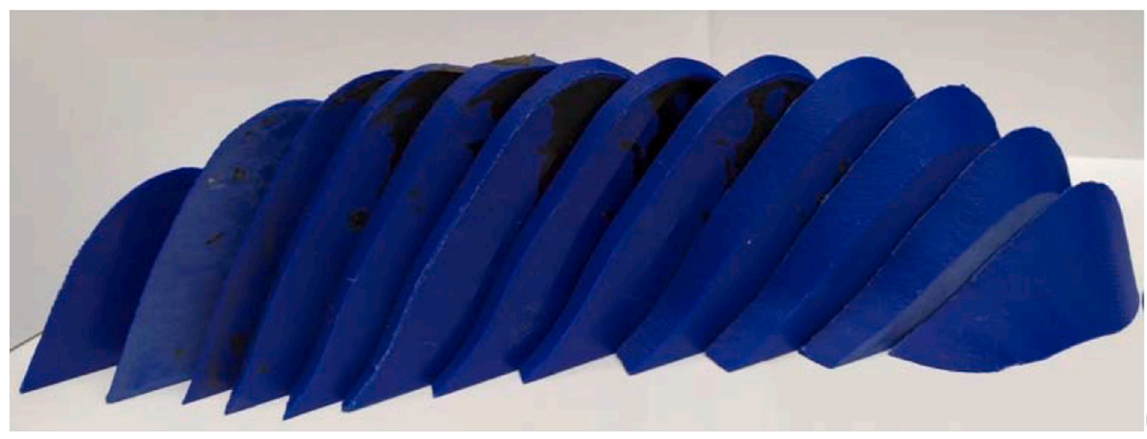

Fig. 17. 3D printed breast phantom. (a) Twelve 10$\mathrm{mm}$ thick slices reproducing the inner part of the breast (adipose + glandular tissues). The slices can be compacted to assemble a breast volume, to be inserted in a 3D-printed skin envelope shown on the right of figure (b). The skin envelope is printed with PVA. The whole inner breast phantom printed in Nylon and ABS (glandular tissue and adipose tissue respectively) is shown on the left in (b). [Reprinted from [91] with the permission of the publisher].

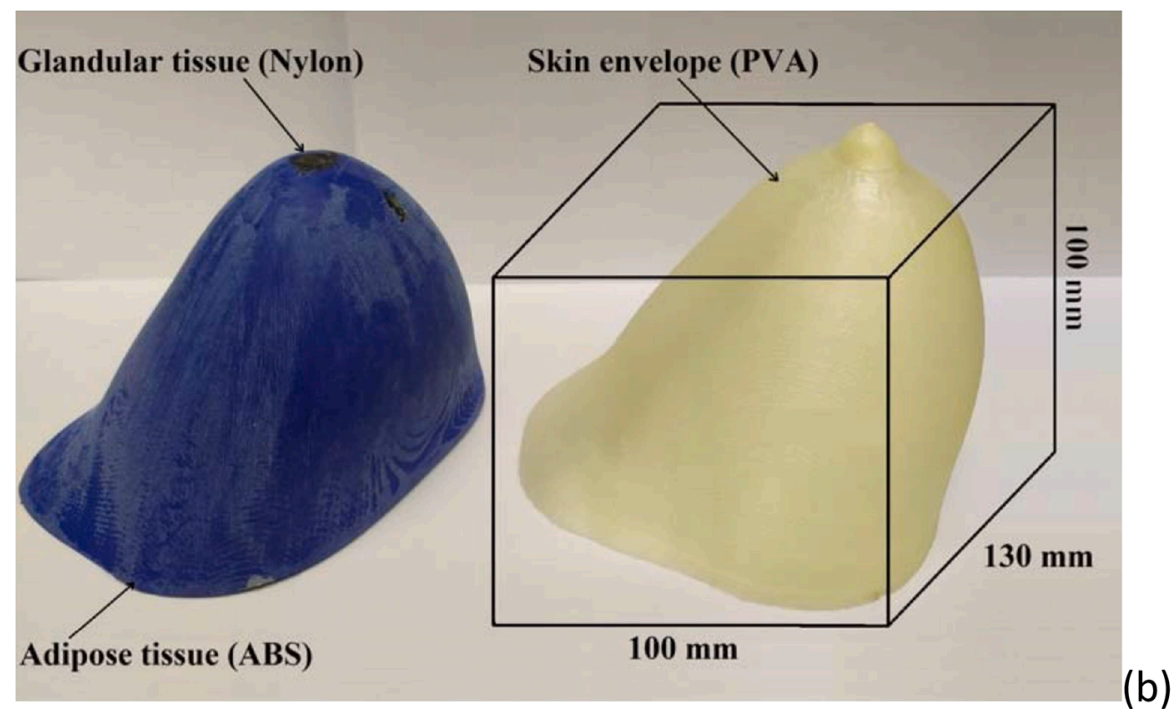

printing paper and a conventional inkjet printer [49]. Several x-ray contrast substances such as potassium iodine, titanium oxide, radiology contrast can be used as ink additives. Fig. 18 shows a phantom produced from a $70 \mu \mathrm{m}$ thick parching paper [49]. The parchment paper, which costs about $80 \$$, was studied by the authors who found to have similar $\mathrm{x}$ ray attenuation properties as the adipose tissue. The physical phantom is fabricated from the virtual digital model, reported by Graff et al. [47] and discussed in Section III, in a slice by slice through inkjet printing, using the parchment paper and a radiopaque ink containing 33\% (I33\%) or $25 \%$ (I25\%) iohexol by volume. This approach for producing anthropomorphic phantoms has an advantage of low price as they are produced with commercially available inkjet printers, low-cost ink enhancers and some cheap additional materials. The inkjet printer in this study is commercially available as a desktop inkjet printer (Epson Workforce 630) which costs about 60\$. Reusable cartridges are used instead of the original manufacturer's cartridges, which cost about $4 \$$ each. Because the printer has several color cartridges, it is possible to print additional "fabrics" in different "colors." For example, to print the

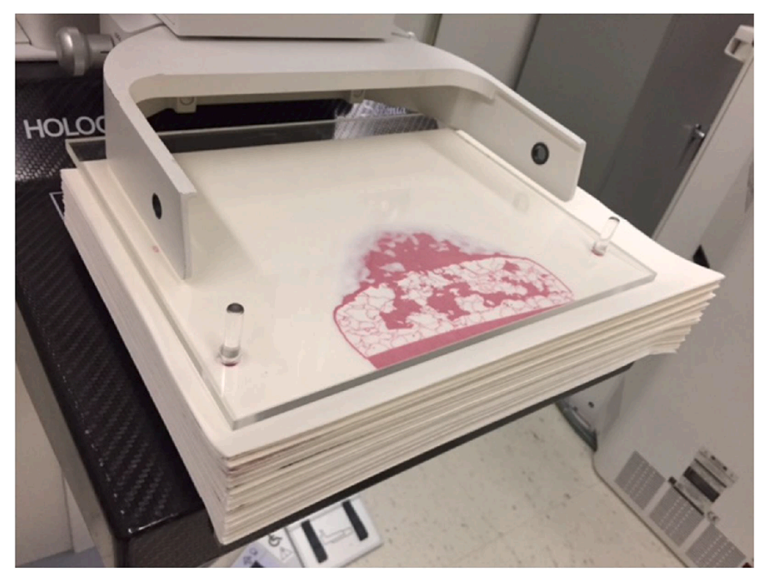

(a)

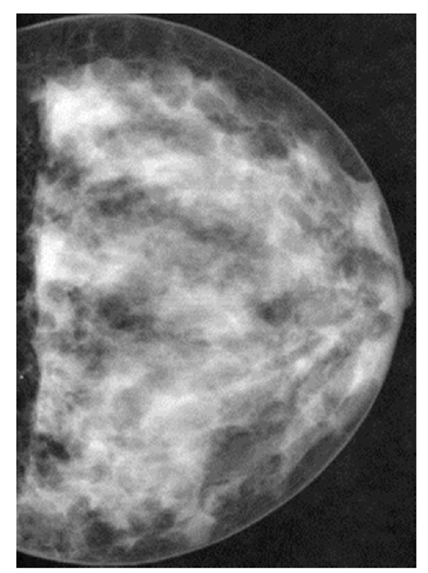

(b)

Fig. 18. Breast physical phantom built from parchment paper: (a) paper phantom and (b) its mammography image [Reprinted from [49] with the permission of the publisher]. 
skin, an appropriate concentration of iodine ink can be determined and placed in a separate cartridge. Different colors can then be applied to the skin and glandular tissue of the images and printed accordingly. The number of materials that can be printed at once is not limited to the number of color cartridges available on the printer, as it is possible to combine primary colors at different levels to create multiple colors. Limitation is related to the use of iodine as a dopant, which limits its use for applications under $33 \mathrm{keV}$.

\section{Conclusions}

This paper presents a brief historical tour of the development and use of anthropomorphic breast models dedicated to x-ray breast imaging. The revealed approaches in creation of such phantoms showed their advantages and limitations, as well as resources needed to be used. Depending on the specific x-ray breast application, one or another approach may be preferred. While the computational phantoms are quite advanced in terms of realistic breast anatomy modelling, the production of physical anthropomorphic models is still a challenge. The available manufacturing techniques for the production of physical phantoms, are still expensive (as expensive and time-consuming task) and may work with a limited selection of materials, only. Although a lot of work has been performed in this area, there is a huge need of novel materials exhibiting similar $\mathrm{x}$-ray absorption coefficients to these of the different breast tissue types for the typical energy range used in mammography, as well as there is a need for low cost production technologies. These are major challenges that the fabrication of anthropomorphic breast phantoms for $\mathrm{x}$-ray imaging needs to face.

\section{Acknowledgement}

This review article is supported by the Bulgarian National Science Fund under grant agreement DN17/2.

\section{References}

[1] Badano A, Badal A, Glick S, Graff CG, Samuelson F, Sharma D, et al. In silico imaging clinical trials for regulatory evaluation: Initial considerations for VICTRE, a demonstration study. Progress in Biomedical Optics and Imaging Proceedings of SPIE2017 DOI: 10.1117/12.2255746.

[2] Badano A, Graff CG, Badal A, Sharma D, Zeng R, Samuelson FW, et al. Evaluation of digital breast tomosynthesis as replacement of full-field digital mammography using an in silico imaging trial. JAMA Netw Open. 2018;1:e185474. https://doi org/10.1001/jama network open.2018.54742717000 [pii].

[3] Sharma D, Graff CG, Badal A, Zeng R, Sawant P, Sengupta A, et al. Technical Note: In silico imaging tools from the VICTRE clinical trial. Med Phys 2019;46: 3924-8. https://doi.org/10.1002/mp.13674.

[4] Mettivier G, Sarno A, Boone JM, Bliznakova K, di Franco F, Russo P. Virtual clinical trials in $3 \mathrm{D}$ and 2D breast imaging with digital phantoms derived from clinical breast CT scans. In: Chen GH, Bosmans H, editors.: SPIE; 2020 DOI: $10.1117 / 12.2548224$.

[5] Bliznakova K, Buliev I, Bliznakov Z. Anthropomorphic phantoms in image quality and patient dose optimization. A EUTEMPE Network book: IOP Publishing 2018. https://doi.org/10.1088/2053-2563/aae197.

[6] Kramer R, Zankl M, Williams G, Drexler G. Part I: The male (ADAM) and female (EVA) adult mathematical phantoms. Gesellschaft für Strahlenund Umweltforschung; Munich: 1982. The calculation of dose from external photon exposures using reference human phantoms and Monte Carlo methods. GSFBericht-S-885; 1982.

[7] Cristy M. Mathematical phantoms representing children of various ages for use in estimates of internal dose. US Nuclear Regulatory Commission Rep NUREG/CR1159 (also Oak Ridge National Laboratory Rep ORNL/NUREG/TM-367)1980.

[8] Snyder WS, Ford MR, G. WG. Estimates of Specific Absorbed Fractions for Monoenergetic Photon Sources Uniformly Distributed in Various Organs of a Heterogeneous Phantom: New York, NY; 1978.

[9] De Paredes ES. Atlas of film-screen mammography/Ellen Shaw de Paredes. United States: Baltimore : Urban \& Schwarzenberg, c1989; 1989.

[10] Novak R. Transformation of the female breast during compression at mammography with special reference to the importance for localization of a lesion. Acta Radiol Suppl 1988;371:1-47.

[11] Bliznakova K, Bliznakov Z, Bravou V, Kolitsi Z, Pallikarakis N. A threedimensional breast software phantom for mammography simulation. Phys Med Biol 2003;48:3699-719. https://doi.org/10.1088/0031-9155/48/22/006.
[12] Bakic PR, Albert M, Brzakovic D, Maidment AD. Mammogram synthesis using a three-dimensional simulation. III. Modeling and evaluation of the breast ductal network. Med Phys 2003;30:1914-25. https://doi.org/10.1118/1.1586453.

[13] Bakic PR, Albert M, Brzakovic D, Maidment AD. Mammogram synthesis using a 3D simulation. I. Breast tissue model and image acquisition simulation. Med Phys 2002;29:2131-9. https://doi.org/10.1118/1.1501143.

[14] Bakic PR, Albert M, Brzakovic D, Maidment AD. Mammogram synthesis using a 3D simulation. II. Evaluation of synthetic mammogram texture. Med Phys 2002; 29:2140-51. https://doi.org/10.1118/1.1501144.

[15] Bakic PR, Zhang C, Maidment AD. Development and characterization of an anthropomorphic breast software phantom based upon region-growing algorithm. Med Phys 2011;38:3165-76. https://doi.org/10.1118/1.3590357.

[16] Pokrajac DD, Maidment AD, Bakic PR. Optimized generation of high resolution breast anthropomorphic software phantoms. Med Phys 2012;39:2290-302. https://doi.org/10.1118/1.3697523.

[17] Lau BA, Reiser I, Nishikawa RM, Bakic PR. A statistically defined anthropomorphic software breast phantom. Med Phys 2012;39:3375-85. https:// doi.org/10.1118/1.4718576.

[18] Chen FY, Pokrajac D, Shi XQ, Liu FS, Maidment ADA, Bakic PR. Partial Volume Simulation in Software Breast Phantoms. Medical Imaging 2012: Physics of Medical Imaging. 2012;8313:Artn 83134u DOI: https://doi.org/10.1117/ 12.912242 .

[19] Chen FY, Bakic PR, Maidment ADA, Jensen ST, Shi XQ, Pokrajac DD. Description and characterization of a novel method for partial volume simulation in software breast phantoms. IEEE Trans Med Imaging 2015;34:2146-61. https://doi.org/ 10.1109/Tmi.2015.2424854.

[20] Bakic PR, Lau B, Carton AK, Reiser I, Maidment ADA, Nishikawa RM. An anthropomorphic software breast phantom for tomosynthesis simulation: Power spectrum analysis of phantom projections. In: Lecture Notes in Computer Science (including subseries Lecture Notes in Artificial Intelligence and Lecture Notes in Bioinformatics); 2010. p. 452-8. https://doi.org/10.1007/978-3-642-13666-5_ 61.

[21] Cockmartin L, Bakic PR, Bosmans H, Maidment ADA, Gall H, Zerhouni M, et al. Power spectrum analysis of an anthropomorphic breast phantom compared to patient data in 2D digital mammography and breast tomosynthesis. In: Lecture Notes in Computer Science (including subseries Lecture Notes in Artificial Intelligence and Lecture Notes in Bioinformatics); 2014. p. 423-9. https://doi. org/10.1007/978-3-319-07887-8_59.

[22] Dance DR, Hunt RA, Bakic PR, Maidment ADA, Sandborg M, Ullman G, et al. Breast dosimetry using high-resolution voxel phantoms. Radiat Prot Dosim 2005; 114:359-63. https://doi.org/10.1093/rpd/nch510.

[23] Hunt RA, Dance DR, Bakic PR, Maidment ADA, Sandborg M, Ullman G, et al. Calculation of the properties of digital mammograms using a computer simulation. Radiat Prot Dosim 2005;114:395-8. https://doi.org/10.1093/rpd/ nch519.

[24] Tischenko O, Hoeschen C, Dance DR, Hunt RA, Maidment ADA, Bakic PR. Evaluation of a novel method of noise reduction using computer-simulated mammograms. Radiat Prot Dosim 2005;114:81-4. https://doi.org/10.1093/rpd/ nch517.

[25] Richard FJP, Bakić PR, Maidment ADA. Mammogram registration: A phantombased evaluation of compressed breast thickness variation effects. IEEE Trans Med Imaging 2006;25:188-97. https://doi.org/10.1109/tmi.2005.862204.

[26] Kontos D, Bakic PR, Maidment ADA. Analysis of parenchymal texture properties in breast tomosynthesis images. Progress in Biomedical Optics and Imaging Proceedings of SPIE. PART 1 ed2007 DOI: 10.1117/12.713851.

[27] Kontos D, Ikejimba LC, Bakic PR, Troxel AB, Conant EF, Maidment ADA. Analysis of parenchymal texture with digital breast tomosynthesis: Comparison with digital mammography and implications for cancer risk assessment. Radiology 2011;261:80-91. https://doi.org/10.1148/radiol.11100966.

[28] Young S, Bakic PR, Myers KJ, Jennings RJ, Park S. A virtual trial framework for quantifying the detectability of masses in breast tomosynthesis projection data. Med Phys 2013;40. https://doi.org/10.1118/1.4800501.

[29] Zeng R, Park S, Bakic P, Myers KJ. Evaluating the sensitivity of the optimization of acquisition geometry to the choice of reconstruction algorithm in digital breast tomosynthesis through a simulation study. Phys Med Biol 2015;60:1259-88. https://doi.org/10.1088/0031-9155/60/3/1259.

[30] Bakic PR, Barufaldi B, Higginbotham D, Weinstein SP, Avanaki AN, Espig KS, et al. Virtual clinical trial of lesion detection in digital mammography and digital breast tomosynthesis. Progress in Biomedical Optics and Imaging - Proceedings of SPIE2018 DOI: 10.1117/12.2294934.

[31] Carton AK, Bakic P, Ullberg C, Derand H, Maidment AD. Development of a physical 3D anthropomorphic breast phantom. Med Phys 2011;38:891-6. https:// doi.org/10.1118/1.3533896.

[32] Bliznakova K, Suryanarayanan S, Karellas A, Pallikarakis N. Evaluation of an improved algorithm for producing realistic 3D breast software phantoms: application for mammography. Med Phys 2010;37:5604-17. https://doi.org/ 10.1118/1.3491812.

[33] Hintsala H, Bliznakova K, Pallikarakis N, Jämsä T. Modelling of irregular breast lesions. 4 ed2009. p. 2024-7 DOI: 10.1007/978-3-642-03882-2-537.

[34] Bliznakova K, Dukov N, Feradov F, Gospodinova G, Bliznakov Z, Russo P, et al. Development of breast lesions models database. Phys Med 2019;64:293-303. https://doi.org/10.1016/j.ejmp.2019.07.017.

[35] Dukov N, Bliznakova K, Feradov F, Buliev I, Bosmans H, Mettivier G, et al. Models of breast lesions based on three-dimensional X-ray breast images. Phys Med 2019; 57:80-7. https://doi.org/10.1016/j.ejmp.2018.12.012. 
[36] Zyganitidis C, Bliznakova K, Pallikarakis N. A novel simulation algorithm for soft tissue compression. Med Biol Eng Comput 2007;45:661-9. https://doi.org/ 10.1007/s11517-007-0205-y.

[37] Imran A, Bakic PR, Pokrajac DD. Spatial distribution of adipose compartments size, shape and orientation in a CT breast image of a mastectomy specimen. 2015 IEEE Signal Processing in Medicine and Biology Symposium (SPMB)2015. p. 1-2 DOI: 10.1109/SPMB.2015.7405460.

[38] Dukov NT, Feradov FN, Gospodinova GD, Bliznakova KS. An approach for printing tissue-mimicking abnormalities dedicated to applications in breast imaging. In: 28th International Scientific Conference Electronics, ET 2019 Proceedings 2019 DOI: 10.1109/et.2019.8878587.

[39] Bliznakova K, Sechopoulos I, Buliev I, Pallikarakis N. BreastSimulator: a software platform for breast X-ray imaging research. J Biomed Graphics Comput 2012;2: 1-14. https://doi.org/10.1088/1361-6560/aa6ca3.

[40] Mettivier G, Bliznakova K, Sechopoulos I, Boone JM, Di Lillo F, Sarno A, et al. Evaluation of the BreastSimulator software platform for breast tomography. Phys Med Biol 2017;62:6446-66. https://doi.org/10.1088/1361-6560/aa6ca3.

[41] Mettivier G, Bliznakova K, Di Lillo F, Sarno A, Russo P. Evaluation of the BreastSimulator software platform for breast tomography: Preliminary results. In Lecture Notes in Computer Science (including subseries Lecture Notes in Artificial Intelligence and Lecture Notes in Bioinformatics); 2016. p. 145-51 DOI: 10.1007/ 978-3-319-41546-8_19.

[42] Bliznakova K, Kolitsi Z, Pallikarakis N. Dual-energy mammography: Simulation studies. Phys Med Biol 2006;51:4497-515. https://doi.org/10.1088/0031-9155/ 51/18/004.

43] Malliori A, Bliznakova K, Sechopoulos I, Kamarianakis Z, Fei B, Pallikarakis N. Breast tomosynthesis with monochromatic beams: A feasibility study using Monte Carlo simulations. Phys Med Biol 2014;59:4681-96. https://doi.org/10.1088/ 0031-9155/59/16/4681.

[44] Liaparinos P, Bliznakova K. Monte Carlo performance on the x-ray converter thickness in digital mammography using software breast models. Med Phys 2012; 39:6638-51. https://doi.org/10.1118/1.4757919.

[45] Bliznakova K, Mettivier G, Russo P, Buliev I. Contrast detail phantoms for X-ray phase-contrast mammography and tomography. Lect Notes Comput Sc. 2016; 9699:611-7. https://doi.org/10.1007/978-3-319-41546-8_76.

[46] Feradov F, Marinov S, Bliznakova K. Physical Breast Phantom Dedicated for Mammography Studies. In: Henriques J, de Carvalho P, Neves N, editors. Springer; 2020. p. 344-52 DOI: 10.1007/978-3-030-31635-8_41.

[47] Graff CG. A New Open-Source, Multi-Modality Digital Breast Phantom. Medical Imaging 2016: Physics of Medical Imaging. 97832016 10.1117/12.2216312 Artn 978309.

[48] Ikejimba LC, Salad J, Graff CG, Ghammraoui B, Cheng WC, Lo JY, et al. A four alternative forced choice (4AFC) methodology for evaluating microcalcification detection in clinical full-field digital mammography (FFDM) and digital breast tomosynthesis (DBT) systems using an inkjet-printed anthropomorphic phantom. Med Phys 2019;46:3883-92. https://doi.org/10.1002/mp.13629.

[49] Ikejimba LC, Graff CG, Rosenthal S, Badal A, Ghammraoui B, Lo JY, et al. A nove physical anthropomorphic breast phantom for 2D and 3D x-ray imaging. Med Phys 2017;44:407-16. https://doi.org/10.1002/mp.12062.

[50] Sauer TJ, Graff CG, Zeng R, Santana M, Sturgeon GM, Bosmans H, et al. Detectability of artificial lesions in anthropomorphic virtual breast phantoms of variable glandular fraction. Prog Biomed Opt Imaging - Proc SPIE 2017. https:// doi.org/10.1117/12.2255896.

[51] Ikejimba LC, Salad J, Graff CG, Goodsitt M, Chan HP, Zhao W, et al. Assessment of task-based performance from five clinical DBT systems using an anthropomorphic breast phantom. Proc SPIE - Int Soc Opt Eng 2020 DOI: 10.1117/12.2564357.

[52] Elangovan P, Mackenzie A, Dance DR, Young KC, Cooke V, Wilkinson L, et al. Design and validation of realistic breast models for use in multiple alternative forced choice virtual clinical trials. Phys Med Biol 2017;62:2778-94. https://doi org/10.1088/1361-6560/aa622c.

[53] Elangovan P, Alrehily F, Pinto RF, Rashidnasab A, Dance DR, Young KC, et al. Simulation of spiculated breast lesions. Prog Biomed Opt Imaging - Proc SPIE 2016 DOI: $10.1117 / 12.2216227$.

[54] Rashidnasab A, Elangovan P, Diaz O, Mackenzie A, Young K, Dance D, et al. Simulation of 3D DLA masses in digital breast tomosynthesis. Prog Biomed Opt Imaging - Proc SPIE 2013 DOI: 10.1117/12.2008333.

[55] Elangovan P, Mackenzie A, Dance DR, Young KC, Wells K. Lesion detectability in 2D-mammography and digital breast tomosynthesis using different targets and observers. Phys Med Biol 2018;63. https://doi.org/10.1088/1361-6560/aabd53.

[56] Hadjipanteli A, Elangovan P, Mackenzie A, Wells K, Dance DR, Young KC. The threshold detectable mass diameter for 2D-mammography and digital breast tomosynthesis. Phys Med 2019;57:25-32. https://doi.org/10.1016/j. ejmp.2018.11.014.

[57] MacKenzie A, Kaur S, Elangovan P, Dance DR, Young KC. Comparison of synthetic 2D images with planar and tomosynthesis imaging of the breast using a virtual clinical trial. Prog Biomed Opt Imaging - Proc SPIE 2018 DOI: 10.1117/ 12.2293070

[58] Kazemi S, Diaz O, Elangovan P, Wells K, Lohstroh A. Comparison of three breast imaging techniques using 4-AFC human observation study. Prog Biomed Opt Imag - Proc SPIE 2018 DOI: 10.1117/12.2293201.

[59] Li ZJ, Desolneux A, Muller S, Carton AK. A novel 3D stochastic solid breast texture model for X-Ray breast imaging. Lect Notes Comput Sci 2016;9699: 660-7. https://doi.org/10.1007/978-3-319-41546-8 82.

[60] Youn H, Han JC, Cho MK, Jang SY, Kim HK, Kim JH, et al. Numerical generation of digital mammograms considering imaging characteristics of an imager. Nucl Instrum Meth A. 2011;652:810-4. https://doi.org/10.1016/j.nima.2010.09.088.
[61] Chen B, Shorey J, Saunders Jr RS, Richard S, Thompson J, Nolte LW, et al. An anthropomorphic breast model for breast imaging simulation and optimization. Acad Radiol 2011;18:536-46. https://doi.org/10.1016/j.acra.2010.11.009 S1076-6332(10)00630-6 [pii].

[62] Ma AK, Gunn S, Darambara DG. Introducing DeBRa: a detailed breast model for radiological studies. Phys Med Biol 2009;54:4533-45. https://doi.org/10.1088/ 0031-9155/54/14/010.

[63] Mahr DM, Bhargava R, Insana MF. Three-dimensional in silico breast phantoms for multimodal image simulations. IEEE Trans Med Imaging 2012;31:689-97. https://doi.org/10.1109/TMI.2011.2175401.

[64] Abbey CK, Bakic PR, Pokrajac DD, Maidment ADA, Eckstein MP, Boone JM. Evaluation of non-Gaussian statistical properties in virtual breast phantoms. J Med Imaging (Bellingham). 2019;6:025502. https://doi.org/10.1117/1. JMI.6.2.025502 19014R [pii].

[65] Cockmartin L, Bosmans H, Bliznakova K, Pokrajac D, Imran A, Marshall N, et al. Structured backgrounds using adipose compartment models in a test object for breast imaging performance analysis. In: RSNA, Annual Meeting of the Radiological Society of North America. Chicago, 25-Nov-2016 to 02-Dec20162016.

[66] Imran AA, Pokrajac DD, Maidment ADA, Bakic PR. Estimation of adipose compartment volumes in CT images of a mastectomy specimen. Med Imag 2016: Phys Med Imag. 2016;9783:Artn 97832o DOI: 10.1117/12.2217175.

[67] Imran AAZ, Bakic PR, Pokrajac DD. Spatial distribution of adipose compartments size, shape and orientation in a CT breast image of a mastectomy specimen. In: IEEE Signal Processing in Medicine and Biology Symposium - Proceedings; 2015 DOI: $10.1109 / \mathrm{spmb} .2015 .7405460$.

[68] Hoeschen C, Fill U, Zankl M, Panzer W, Regulla D, Dohring W. A high-resolution voxel phantom of the breast for dose calculations in mammography. Radiat Prot Dosimetry 2005;114:406-9. https://doi.org/10.1093/rpd/nch558.

[69] Li CM, Segars WP, Tourassi GD, Boone JM, Dobbins 3rd JT. Methodology for generating a 3D computerized breast phantom from empirical data. Med Phys 2009;36:3122-31. https://doi.org/10.1118/1.3140588.

[70] Hsu CM, Palmeri ML, Segars WP, Veress AI, Dobbins 3rd JT. Generation of a suite of 3D computer-generated breast phantoms from a limited set of human subject data. Med Phys 2013;40:043703. https://doi.org/10.1118/1.4794924.

[71] Hsu CM, Palmeri ML, Segars WP, Veress AI, Dobbins 3rd JT. An analysis of the mechanical parameters used for finite element compression of a high-resolution 3D breast phantom. Med Phys 2011;38:5756-70. https://doi.org/10.1118/ 1.3637500 .

[72] Huang SY, Boone JM, Yang K, Packard NJ, McKenney SE, Prionas ND, et al. The characterization of breast anatomical metrics using dedicated breast CT. Med Phys 2011;38:2180-91.

[73] Segars WP, Veress AI, Wells JR, Sturgeon GM, Kiarashi N, Lo JY, et al. Population of 100 Realistic, Patient-Based Computerized Breast Phantoms for Multi-modality Imaging Research. Med Imaging 2014: Phys Med Imaging. 2014;9033:Artn 90331x DOI: $10.1117 / 12.2043868$.

[74] Erickson DW, Wells JR, Sturgeon GM, Samei E, Dobbins JT, Segars WP, et al. Population of 224 realistic human subject-based computational breast phantoms. Med Phys. 2016;43:23-32. https://doi.org/10.1118/1.4937597.

[75] di Franco F, Sarno A, Mettivier G, Hernandez AM, Bliznakova K, Boone JM, et al. GEANT4 Monte Carlo simulations for virtual clinical trials in breast X-ray imaging: Proof of concept. Phys Med 2020;74:133-42. https://doi.org/10.1016/j. ejmp.2020.05.007.

[76] Sarno A, Mettivier G, Di Lillo F, Bliznakova K, Sechopoulos I, Russo P. Homogeneous vs. patient specific breast models for Monte Carlo evaluation of mean glandular dose in mammography. Phys Med. 2018;51:56-63 DOI: S11201797(18)30451-4 [pii] 10.1016/j.ejmp.2018.04.392.

[77] Sarno A, Mettivier G, Tucciariello RM, Bliznakova K, Boone JM, Sechopoulos I, et al. Monte Carlo evaluation of glandular dose in cone-beam X-ray computed tomography dedicated to the breast: Homogeneous and heterogeneous breast models. Phys Med. 2018;51:99-107. S1120-1797(18)30477-0 [pii] 10.1016/j. ejmp. 2018.05.021.

[78] Sarno A, Mettivier G, Di Lillo F, Tucciariello RM, Bliznakova K, Russo P. Normalized glandular dose coefficients in mammography, digital breast tomosynthesis and dedicated breast CT. Phys Med. 2018;55:142-8. S1120-1797 (18)31163-3 [pii] 10.1016/j.ejmp.2018.09.002.

[79] Mettivier G, Sarno A, Boone J, Bliznakova K, di Franco F, Russo P. Virtual clinical trials in $3 \mathrm{D}$ and $2 \mathrm{D}$ breast imaging with digital phantoms derived from clinical breast CT scans. SPIE 2020.

[80] García E, Fedon C, Caballo M, Martí R, Sechopoulos I, Diaz O. Realistic compressed breast phantoms for medical physics applications. In: Bosmans $\mathrm{H}$, Marshall N, Van Ongeval C, editors. 15th International Workshop on Breast Imaging, IWBI 2020: SPIE; 2020 DOI: 10.1117/12.2564273.

[81] Ivanov D, Bliznakova K, Buliev I, Popov P, Mettivier G, Russo P, et al. Suitability of low density materials for 3D printing of physical breast phantoms. Phys Med Biol. 2018;63:175020. https://doi.org/10.1088/1361-6560/aad315.

[82] Santos JC, Almeida CD, Iwahara A, Peixoto JE. Characterization and applicability of low-density materials for making 3D physical anthropomorphic breas phantoms. Radiat Phys Chem. 2019;164:ARTN 108361 DOI: 10.1016/j. radphyschem.2019.108361.

[83] Esposito G, Mettivier G, Bliznakova K, Bliznakov Z, Bosmans H, Bravin A, et al. Investigation of the refractive index decrement of 3D printing materials for manufacturing breast phantoms for phase contrast imaging. Phys Med Biol 2019; 64. https://doi.org/10.1088/1361-6560/ab0670. 
[84] Hernandez AM, Seibert JA, Nosratieh A, Boone JM. Generation and analysis of clinically relevant breast imaging x-ray spectra. Med Phys 2017;44:2148-60. https://doi.org/10.1002/mp.12222.

[85] Mainprize J, Mawdsley G, Carton A-K, Li Z, Klausz R, Muller S, et al. Full-size anthropomorphic phantom for $2 \mathrm{D}$ and $3 \mathrm{D}$ breast x-ray imaging. In: 15th International Workshop on Breast Imaging (IWBI2020). 2020;11513:1151302 DOI: https://doi.org/10.1117/12.2560358.

[86] Kiarashi N, Nolte AC, Sturgeon GM, Segars WP, Ghate SV, Nolte LW, et al. Development of realistic physical breast phantoms matched to virtual breast phantoms based on human subject data. Med Phys 2015;42:4116-26. https://doi. org/10.1118/1.4919771.

[87] Oyiwoja Okoh F, Ahmad Kabir N, Mohd Yusof MF, Azizah Abdullah SN. Measurement of mass attenuation coefficient of polyvinyl alcohol (PVAL) as breast tissue equivalent material in the photon energy range of 16.61-25.26 keV. In: Abdullah M, editor. 2019 International Conference on Semiconductor Materials and Technology, ICoSeMT 2019. 1 ed: Institute of Physics Publishing; 2020 DOI: 10.1088/1742-6596/1535/1/012051.

[88] Prionas ND, Burkett GW, McKenney SE, Chen L, Stern RL, Boone JM. Development of a patient-specific two-compartment anthropomorphic breast phantom. Phys Med Biol 2012;57:4293-307. https://doi.org/10.1088/00319155/57/13/4293.

[89] Sikaria D, Musinksy S, Sturgeon GM, Solomon J, Diao A, Gehm ME, et al. Second generation anthropomorphic physical phantom for mammography and DBT: Incorporating voxelized 3D printing and inkjet printing of iodinated lesion inserts. Medical Imaging 2016: Physics of Medical. Imaging. 2016;9783. https:// doi.org/10.1117/12.2217667. Artn 978360.

[90] Ruvio G, Solimene R, Cuccaro A, Fiaschetti G, Fagan AJ, Cournane S, et al. Multimodal breast phantoms for microwave, ultrasound, mammography, magnetic resonance and computed tomography imaging. Sensors 2020;20. https://doi.org/10.3390/s20082400.

[91] Franco Fd, Mettivier G, Sarno A, Varallo A, Russo P. Manufacturing of physical breast phantoms with 3D printing technology for X-ray breast imaging. In: IEEE Nuclear Science Symposium and Medical Imaging Conference (NSS/MIC); 2019. p. 1-5. https://doi.org/10.1109/nss/mic42101.2019.9059986.

[92] Sage J, Fezzani KL, Fitton I, Hadid L, Moussier A, Pierrat N, et al. Experimental evaluation of seven quality control phantoms for digital breast tomosynthesis. Phys Med 2019;57:137-44. S1120-1797(18)31366-8 [pii] 10.1016/j. ejmp.2018.12.031.

[93] Freed M, Badal A, Jennings RJ, de las Heras H, Myers KJ, Badano A. X-ray properties of an anthropomorphic breast phantom for MRI and x-ray imaging. Phys Med Biol. 2011;56:3513-33 DOI: 10.1088/0031-9155/56/12/005.

[94] Baldelli P, Phelan N, Egan G. Investigation of the effect of anode/filter materials on the dose and image quality of a digital mammography system based on an amorphous selenium flat panel detector. Br J Radiol. 2010;83:290-5. https://doi org $/ 10.1259 / \mathrm{bjr} / 60404532$.

[95] Park S, Jennings R, Liu HM, Badano A, Myers K. A statistical, task-based evaluation method for three-dimensional $\mathrm{x}$-ray breast imaging systems using variable-background phantoms. Med Phys. 2010;37:6253-70. Doi 10.1118/ 1.3488910 .

[96] Taibi A, Fabbri S, Baldelli P, di Maggio C, Gennaro G, Marziani M, et al. Dualenergy imaging in full-field digital mammography: a phantom study. Phys Med Biol 2003;48:1945-56. https://doi.org/10.1088/0031-9155/48/13/307.

[97] Cockmartin L, Marshall N, Bosmans H. Design and evaluation of a phantom with structured background for digital mammography and breast tomosynthesis. Philadelphia, PA2012. p. 642-9 DOI: 10.1007/978-3-642-31271-7_83.

[98] Gang GJ, Tward DJ, Lee J, Siewerdsen JH. Anatomical background and generalized detectability in tomosynthesis and cone-beam CT. Med Phys. 2010; 37:1948-65. https://doi.org/10.1118/1.3352586.

[99] Cockmartin L, Marshall N, Bosmans H. Design and evaluation of a phantom with structured background for digital mammography and breast tomosynthesis. Lecture Notes in Computer Science (including subseries Lecture Notes in Artificial Intelligence and Lecture Notes in Bioinformatics) 2012. p. 642-9 DOI: 10.1007/ 978-3-642-31271-7_83.

[100] Baneva Y, Bliznakova K, Cockmartin L, Marinov S, Buliev I, Mettivier G, et al. Evaluation of a breast software model for 2D and 3D X-ray imaging studies of the breast. Phys Med 2017;41:78-86. https://doi.org/10.1016/j.ejmp.2017.04.024.

[101] Bliznakova K. Development of breast software phantom dedicated for research and educational purposes. Radiation and Applications in Physics, Chemistry, Biology, Medical Sciences, Engineering and Environmental Sciences. 2016;2: 14-9. https://doi.org/10.21175/RadJ.2017.01.004.
[102] Marinov S, Carton AK, Cockmartin L, Marshall NW, Van Ongeval C, Sanchez De La Rosa R, et al. Evaluation of the visual realism of breast texture phantoms in digital mammography. Proc SPIE - Int Soc Opt Eng 2020 DOI: 10.1117/ 12.2564124 .

[103] Bliznakova K, Mettivier G, Russo P, Bliznakov Z. Validation of a software platform for 2D and 3D phase contrast imaging: Preliminary subjective evaluation. Proc SPIE - Int Soc Opt Eng 2020. https://doi.org/10.1117/12.2564356.

[104] Bliznakova K, Russo P, Mettivier G, Requardt H, Popov P, Bravin A, et al. A software platform for phase contrast x-ray breast imaging research. Comput Biol Med 2015;61:62-74. https://doi.org/10.1016/j.compbiomed.2015.03.017.

[105] Petrov D, Marshall NW, Young KC, Bosmans H. Systematic approach to a channelized Hotelling model observer implementation for a physical phantom containing mass-like lesions: Application to digital breast tomosynthesis. Phys Med 2019;58:8-20. S1120-1797(18)31373-5 [pii] 10.1016/j.ejmp.2018.12.033.

[106] Cockmartin L, Bosmans H, Bliznakova K, Pokrajac D, Imran A-A-Z, Marshall N, et al. Creation of Realistic Structured Backgrounds using Adipose Compartment Models in a Test Object for Breast Imaging Performance Analysis. In: Radiological Society of North America (RSNA) 2016, Scientific Assembly and Annual Meeting; 2016. p. 102.

[107] Salomon E, Semturs F, Unger E, Cockmartin L, Petrov D, Vancoillie L, et al. Equivalent breast thickness and dose sensitivity of a next iteration 3D structured breast phantom with lesion models. Proc. SPIE 11312, Medical Imaging 2020: Physics of Medical Imaging 2000 113120K. https://doi.org/10.1117/1 2.2548956 .

[108] Mainprize J, Carton A-K, Klausz R, Li Z, Hunter D, Mawdsley G, et al. Development of a physical 3D anthropomorphic breast texture model using selective laser sintering rapid prototype printing. SPIE Medical Imaging 2018: Physics of Medical Imaging 2018;10573:105730A DOI: https://doi.org/10.111 7/12.2293560.

[109] Schopphoven S, Cavael P, Bock K, Fiebich M, Mader U. Breast phantoms for 2D digital mammography with realistic anatomical structures and attenuation characteristics based on clinical images using 3D printing. Phys Med Biol 2019; 64:215005. https://doi.org/10.1088/1361-6560/ab3f6a.

[110] Badal A, Clark M, Ghammraoui B. Reproducing two-dimensional mammograms with three-dimensional printed phantoms. J Med Imaging (Bellingham) 2018;5: 033501. https://doi.org/10.1117/1.JMI.5.3.033501 17353PRR [pii].

[111] Clark M, Ghammraoui B, Badal A. Reproducing 2D Breast Mammography Images with 3D Printed Phantoms. Medical Imaging 2016: Physics of Medical Imaging. 2016;9783:Artn 97830b DOI: https://doi.org/10.1117/12.2217215.

[112] Mäder U, Fiebich M, Bock K, Schopphoven S. Concept to extend anthropomorphic breast phantoms for 2D digital mammography with movable lesions at variable reproducible positions. In: Fifteenth International Workshop on Breast Imaging. 2020;11513:115131L (2020) DOI: https://doi.org/10.1117/12.2560619.

[113] Okkalidis N. A novel 3D printing method for accurate anatomy replication in patient-specific phantoms. Med Phys 2018;45:4600-6. https://doi.org/10.1002/ mp.13154.

[114] Daskalov S, Okkalidis N, Boone JM, Marinov S, Bliznakov Z, Mettivier G, et al. Anthropomorphic Physical Breast Phantom Based on Patient Breast CT Data: Preliminary Results. In: Henriques J, de Carvalho P, Neves N, editors.: Springer; 2020. p. 367-74 DOI: 10.1007/978-3-030-31635-8_44.

[115] Lindfors KK, Boone JM, Nelson TR, Yang K, Kwan ALC, Miller DF. Dedicated breast CT: Initial clinical experience. Radiology 2008;246:725-33. DOI 10.1148/ radiol.2463070410.

[116] Burgess AE, Judy PE. Signal detection in power-law noise: Effect of spectrum exponents. J Opt Soc Am A: Opt Image Sci Vis 2007;24:B52-60. https://doi.org/ 10.1364/josaa.24.000b52.

[117] Burgess AE, Jacobson FL, Judy PF. Human observer detection experiments with mammograms and power-law noise. Med Phys 2001;28:419-37. https://doi.org/ 10.1118/1.1355308.

[118] Rossman AH, Catenacci M, Zhao C, Sikaria D, Knudsen JE, Dawes D, et al. Threedimensionally-printed anthropomorphic physical phantom for mammography and digital breast tomosynthesis with custom materials, lesions, and uniform quality control region. J Med Imaging 2019;6. https://doi.org/10.1117/1. jmi.6.2.021604.

[119] Mettivier G, Sarno A, Franco Fd, Bliznakova K, Bliznakov Z, Hernandez AM, et al. The Napoli-Varna-Davis project for virtual clinical trials in X-ray breast imaging. In: 2019 IEEE Nuclear Science Symposium and Medical Imaging Conference (NSS/MIC)2019. p. 1-5 DOI: 10.1109/nss/mic42101.2019.9059828. 